\title{
Collective instabilities and beam-plasma interactions in intense heavy ion beams
}

\author{
Ronald C. Davidson, Igor Kaganovich, Hong Qin, and Edward A. Startsev \\ Princeton Plasma Physics Laboratory, Princeton, New Jersey, USA \\ Dale R. Welch and David V. Rose \\ Mission Research Corporation, Albuquerque, New Mexico, USA
}

Han S. Uhm

Ajou University, Suwon, Korea

(Received 12 May 2004; published 17 November 2004)

\begin{abstract}
This paper presents a survey of the present theoretical understanding of collective processes and beam-plasma interactions affecting intense heavy ion beam propagation in heavy ion fusion systems. In the acceleration and beam transport regions, the topics covered include discussion of the conditions for quiescent beam propagation over long distances; the electrostatic Harris-type instability and the transverse electromagnetic Weibel-type instability in strongly anisotropic, one-component non-neutral ion beams; and the dipole-mode, electron-ion two-stream instability driven by an (unwanted) component of background electrons. In the plasma plug and target chamber regions, collective processes associated with the interaction of the intense ion beam with a charge-neutralizing background plasma are described, including the electrostatic electron-ion two-stream instability, the electromagnetic Weibel instability, and the resistive hose instability. Operating regimes are identified where the possible deleterious effects of collective processes on beam quality are minimized.
\end{abstract}

DOI: $10.1103 /$ PhysRevSTAB.7.114801

PACS numbers: 52.40.Mj, 52.58.Hm, 29.27.Bd, 52.35.Qz

\section{INTRODUCTION}

High energy ion accelerators, transport systems, and storage rings [1-5] are used for fundamental research in high energy and nuclear physics and for applications such as heavy ion fusion, spallation neutron sources, and nuclear waste transmutation. Charged particle beams are subject to various collective processes that can deteriorate the beam quality. Of particular importance at the high beam currents and charge densities of interest for heavy ion fusion are the effects of the intense self-fields produced by the beam space charge and current on determining detailed equilibrium, stability, and transport properties. In general, a complete description of collective processes in intense charged particle beams is provided by the nonlinear Vlasov-Maxwell equations [1] for the self-consistent evolution of the beam distribution function, $f_{b}(\mathbf{x}, \mathbf{p}, t)$, and the electric and magnetic fields, $\mathbf{E}(\mathbf{x}, t)$ and $\mathbf{B}(\mathbf{x}, t)$. While considerable progress has been made in analytical and numerical simulation studies of intense beam propagation [6-77], the effects of finite geometry and intense self-fields often make it difficult to obtain detailed predictions of beam equilibrium, stability, and transport properties based on the VlasovMaxwell equations. Nonetheless, often with the aid of numerical simulations, there has been considerable recent analytical progress in applying the Vlasov-Maxwell equations to investigate the detailed equilibrium and stability properties of intense charged particle beams. These investigations include a wide variety of collective interaction processes ranging from the electrostatic
Harris instability [30-36] and electromagnetic Weibel instability [37-42] driven by large temperature anisotropy with $T_{\perp b} \gg T_{\| b}$ in a one-component non-neutral ion beam, to wall-impedance-driven collective instabilities [43-45,49], to the dipole-mode two-stream instability for an intense ion beam propagating through a partially neutralizing electron background [46-60], to the resistive hose instability [61-67], the sausage and hollowing instabilities [68-70], and the multispecies Weibel and twostream instabilities [71-73] for an intense ion beam propagating through a background plasma [74-77], to the development of a nonlinear stability theorem $[20,21]$ in the smooth-focusing approximation.

In this paper, we present a brief survey of the present theoretical understanding of collective processes and beam-plasma interactions affecting intense heavy ion beam propagation in heavy ion fusion systems. In the acceleration and beam transport regions, the topics covered in Secs. II and III include discussion of the conditions for quiescent beam propagation over long distances; the electrostatic Harris-type instability and the transverse electromagnetic Weibel-type instability in strongly anisotropic, one-component non-neutral ion beams; and the dipole-mode, electron-ion two-stream instability driven by an (unwanted) component of background electrons. In the plasma plug and target chamber regions, collective processes associated with the interaction of the intense ion beam with a charge-neutralizing background plasma are described in Sec. IV, including the electrostatic electron-ion two-stream instability, the electromagnetic 
Weibel instability, and the resistive hose instability. Operating regimes are identified where the possible deleterious effects of collective processes on beam quality are minimized. Here, "plasma plug" [78-81] refers to a region containing preformed plasma immediately following the final focusing magnets. The plasma volume is sufficiently large that the (mobile) electrons neutralize the ion beam space charge and assist in focusing the ion beam to a small spot size.

To briefly summarize, the present analysis assumes a long charge bunch (bunch length $\ell_{b} \gg$ bunch radius $r_{b}$ ) with directed axial kinetic energy $\left(\gamma_{b}-1\right) m_{b} c^{2}$ propagating in the $z$ direction through a perfectly conducting cylindrical pipe with constant radius $r_{w}$. The analysis is carried out in the smooth-focusing approximation, where the applied transverse focusing force is modeled by $\mathbf{F}_{\text {foc }}=-\gamma_{b} m_{b} \omega_{f}^{2} \mathbf{x}_{\perp}$. Here, $\gamma_{b}=\left(1-\beta_{b}^{2}\right)^{-1 / 2}$ is the relativistic mass factor, $V_{b}=\beta_{b} c$ is the directed axial velocity of the charge bunch, $m_{b}$ is the particle rest mass, $\omega_{f}=$ const is the single-particle oscillation frequency associated with the applied focusing force, and $\mathbf{x}_{\perp}=$ $x \mathbf{e}_{x}+y \mathbf{e}_{y}$ is the transverse displacement of a beam particle from the cylinder axis. Denoting the characteristic number density of beam particles by $\hat{n}_{b}$ and the particle charge by $e_{b}$, it is convenient to introduce the relativistic plasma frequency $\hat{\omega}_{p b}$ defined by $\hat{\omega}_{p b}=$ $\left(4 \pi \hat{n}_{b} e_{b}^{2} / \gamma_{b} m_{b}\right)^{1 / 2}$ and the normalized (dimensionless) beam intensity $s_{b}$ defined by $s_{b}=\hat{\omega}_{p b}^{2} / 2 \gamma_{b}^{2} \omega_{f}^{2}$ [1]. Furthermore, the particle dynamics in the beam frame are assumed to be nonrelativistic.

In the following sections, we give a brief overview of the present understanding of several collective instabilities that can develop in intense charged particle beams. While the summaries presented here are necessarily short, the references in the associated bibliography provide considerable detailed information.

\section{ANISOTROPY-DRIVEN INSTABILITIES IN ONE-COMPONENT BEAMS}

A remarkable feature of intense beam propagation is the existence of a stability theorem based on the nonlinear Vlasov-Maxwell equations [1,20-22]. To summarize, for a long, one-component coasting beam in the smooth-focusing approximation, the stability theorem, expressed in the beam frame $\left(\beta_{b}=0\right.$ and $\left.\gamma_{b}=1\right)$, states that any equilibrium distribution function $f_{b}^{0}(H)$ that satisfies

$$
\frac{\partial}{\partial H} f_{b}^{0}(H) \leq 0
$$

is nonlinearly stable to perturbations with arbitrary polarization [20,21]. Here, $H=\left(p_{r}^{2}+p_{\theta}^{2}+p_{z}^{2}\right) / 2 m_{b}+$ $m_{b} \omega_{f}^{2} r^{2} / 2+e_{b} \phi^{0}(r)$ is the single-particle Hamiltonian in the beam frame, and $\phi^{0}(r)$ is the electrostatic potential determined self-consistently in terms of the beam space charge from Poisson's equation. Therefore, from Eq. (1), any isotropic distribution function that is a monotonic decreasing function of energy in the beam frame is nonlinearly stable. The validity of this stability theorem has been demonstrated in nonlinear perturbative particle simulations [55,82] for intense beam propagation over thousands of equivalent lattice periods.

While Eq. (1) is a sufficient condition for stability, a necessary condition for instability is that the beam distribution function have some nonthermal feature such as an inverted population in phase space [6-8], or a strong energy anisotropy. Energy anisotropies are well known in electrically neutral plasmas to provide the free energy to drive the classical electrostatic Harris instability [30] and the electromagnetic Weibel instability [37]. This anisotropy can be either a temperature anisotropy or an anisotropy in the relative directed kinetic energy of the plasma components.

\section{A. Electrostatic Harris-type instability}

In electrically neutral plasmas with strongly anisotropic distributions $\left(T_{\| b} / T_{\perp b} \ll 1\right)$, collective instabilities may develop if there is sufficient coupling between the transverse and longitudinal degrees of freedom [30,37]. Such anisotropies develop naturally in accelerators [2]. Indeed, due to conservation of energy for particles with charge $e_{b}$ and mass $m_{b}$ accelerated by a voltage $V$, the energy spread of particles in the beam does not change, and (nonrelativistically) $\Delta E_{b i}=m_{b} \Delta v_{b i}^{2} / 2=$ $\Delta E_{b f} \simeq m_{b} V_{b} \Delta v_{b f}$, where $V_{b}=\left(2 e_{b} V / m_{b}\right)^{1 / 2}$ is the average beam velocity after acceleration across a potential difference $V$. Therefore, the longitudinal velocity spread squared, or equivalently the temperature, changes according to $T_{\| b f} \simeq T_{\| b i}^{2} / 2 e_{b} V$ (for a nonrelativistic beam). At the same time, the transverse temperature may increase due to nonlinearities in the applied and self-field forces, nonstationary beam profiles, and beam mismatch. These processes provide the free energy to drive collective instabilities and may lead to a deterioration of beam quality. Such instabilities may also lead to an increase of longitudinal velocity spread, which will make the focusing of the beam difficult and may impose a limit on the minimum spot size achievable in heavy ion fusion experiments.

Recent investigations [31-36] of the Harris-type electrostatic instability [30] in intense one-component beams have focused on analytical studies of linear stability properties and numerical simulations of the nonlinear development. In recent studies [31-33] we have considered electrostatic perturbations $(\nabla \times \delta \mathbf{E} \simeq 0$ and $\delta \mathbf{B} \simeq$ $0)$ about a thermal equilibrium distribution with temperature anisotropy $\left(T_{\perp b}>T_{\| b}\right)$ described in the beam frame $\left(V_{b}=0\right.$ and $\left.\gamma_{b}=1\right)$ by the self-consistent axisymmetric Vlasov equilibrium 


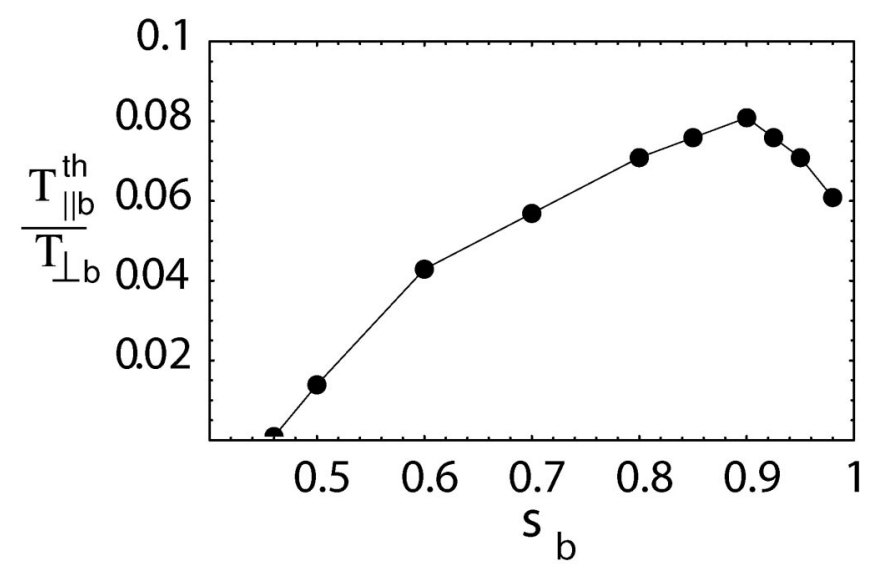

FIG. 1. Longitudinal threshold temperature $T_{\| b}^{\text {th }}$ normalized to the transverse temperature $T_{\perp b}$ for the onset of the electrostatic Harris instability plotted versus normalized beam intensity $s_{b}=\hat{\omega}_{p b}^{2} / 2 \omega_{f}^{2}$.

$$
\begin{aligned}
f_{b}^{0}(r, \mathbf{p})= & \frac{\hat{n}_{b}}{\left(2 \pi m_{b} T_{\perp b}\right)} \exp \left(-\frac{H_{\perp}}{T_{\perp b}}\right) \frac{1}{\left(2 \pi m_{b} T_{\| b}\right)^{1 / 2}} \\
& \times \exp \left(-\frac{p_{z}^{2}}{2 m_{b} T_{\| b}}\right) .
\end{aligned}
$$

Here, $\quad H_{\perp}=p_{\perp}^{2} / 2 m_{b}+(1 / 2) m_{b} \omega_{f}^{2}\left(x^{2}+y^{2}\right)+e_{b} \phi^{0}(r)$ is the single-particle Hamiltonian for the transverse particle motion, $p_{\perp}=\left(p_{r}^{2}+p_{\theta}^{2}\right)^{1 / 2}$ is the transverse particle momentum, $r=\left(x^{2}+y^{2}\right)^{1 / 2}$ is the radial distance from the beam axis, $\omega_{f}=$ const is the transverse focusing frequency, and $\phi^{0}(r)$ is determined self-consistently from Poisson's equation $r^{-1}(\partial / \partial r)\left[r \partial \phi^{0} / \partial r\right]=$ $-4 \pi e_{b} \int d^{3} p f_{b}^{0}(r, \mathbf{p})$. Assuming three-dimensional electrostatic perturbations, an infinite dimension matrix dispersion equation has been derived and the stability results have been compared with numerical simulations using the Beam Equilibrium, Stability and Transport (BEST) nonlinear perturbative particle code [31-33]. The results clearly show that moderately intense beams with normalized intensity parameter $s_{b}=\hat{\omega}_{p b}^{2} / 2 \omega_{f}^{2} \gtrsim 0.5$ are linearly unstable to short-wavelength perturbations with $k_{z}^{2} r_{b}^{2} \gtrsim 1$, provided $T_{\| b} / T_{\perp b}$ is smaller than some threshold value (Fig. 1). Here, $\hat{\omega}_{p b}=\left(4 \pi \hat{n}_{b} e_{b}^{2} / m_{b}\right)^{1 / 2}$ is the onaxis $(r=0)$ plasma frequency in the beam frame. Moreover, the mode structure, growth rate, and conditions for the onset of instability are qualitatively similar to analytical predictions [31-33]. Both the simulations and the analytical theory predict that the dipole mode (azimuthal mode number $m=1$ ) is the most unstable mode. The main saturation mechanism for the instability is the resonant wave-particle interactions that occur during the formation of tails in the axial momentum distribution $F_{b}\left(p_{z}, t\right)=\int d^{2} p_{\perp} d^{3} x f_{b}$ [33]. This is illustrated in Fig. 2, and the corresponding time history of the perturbed density $\delta n_{b}=\int d^{3} p \delta f$ is plotted versus $\omega_{f} t$

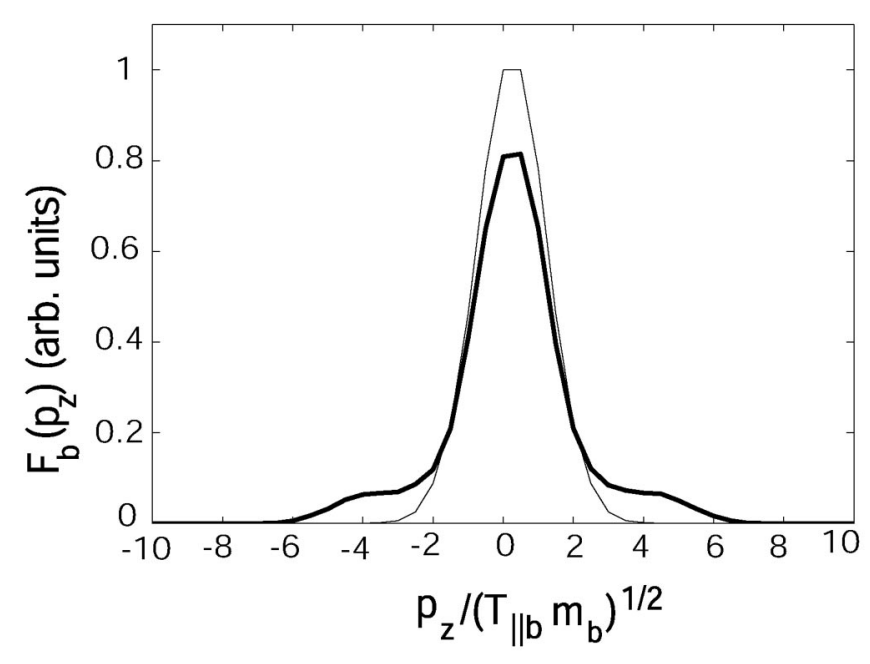

FIG. 2. Plot of average longitudinal momentum distribution $F_{b}\left(p_{z}, t\right)$ at time $t=0$ (thin line) and $t=150 \omega_{f}^{-1}$ (thick line), for normalized beam intensity $s_{b}=0.8$ and $T_{\| b} / T_{\perp b}=0.02$.

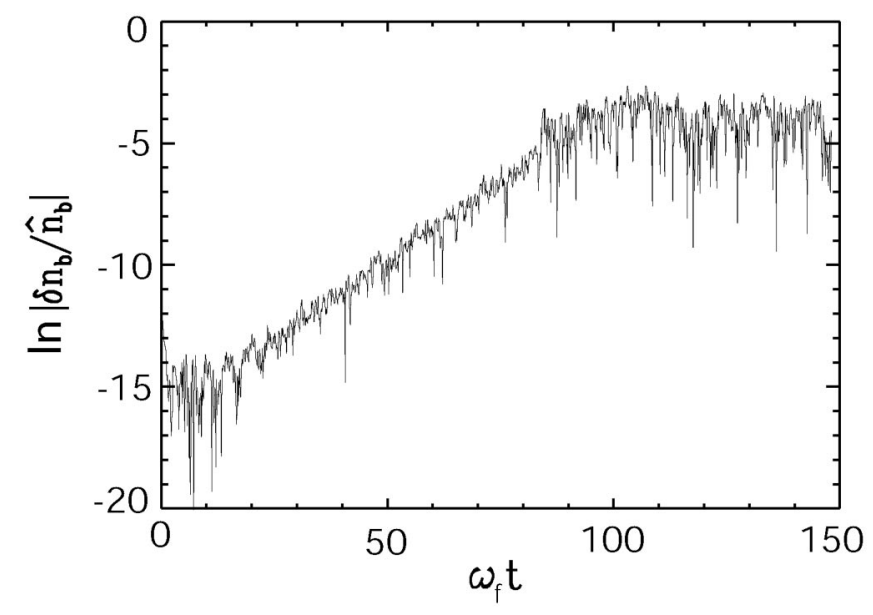

FIG. 3. Time history of the normalized density perturbation $\delta n_{\max } / \hat{n}_{b}$ for $s_{b}=0.8$ and $T_{\| b} / T_{\perp b}=0.02$ at fixed axial position $z$ and radius $r=0.3 r_{b}$ for the same conditions as in Fig. 2.

in Fig. 3 for the case where the initial perturbation has a dominant initial excitation with azimuthal mode number $m=1$ and $k_{z} r_{w}=9$ [33]. During the linear growth stage, note from Fig. 3 that the characteristic instability growth rate is $\operatorname{Im} \omega=0.13 \omega_{f}$. Note also from Fig. 2 that in the nonlinear saturation stage, the total distribution function is still far from equipartitioned, and free energy is still available to drive an instability of the hydrodynamic type [1], or possibly an electromagnetic Weibel-type instability $[37,38]$.

\section{B. Electromagnetic Weibel-type instability}

In multispecies anisotropic beam-plasma systems it is well known that the electromagnetic Weibel instability 
[37-42] can be particularly virulent in affecting the nonlinear dynamics of the system. Such appears not to be the case for an intense one-component charged particle beam [38] because of the strong constraint imposed by the finite transverse geometry and the fact that the Harristype instability described in Sec. II A has a much larger growth rate in the unstable regime.

In a recent calculation [38], we have considered transverse electromagnetic perturbations about the choice of anisotropic equilibrium distribution defined in Eq. (2) in the beam frame. Assuming axisymmetric perturbations $(\partial / \partial \theta=0)$, the perturbed transverse electromagnetic fields are assumed to have polarization $\delta \mathbf{E}^{T}=\delta E_{\theta} \hat{\mathbf{e}}_{\theta}$ and $\delta \mathbf{B}^{T}=\delta B_{r} \hat{\mathbf{e}}_{r}+\delta B_{z} \hat{\mathbf{e}}_{z}$. A linear stability analysis has been carried out based on the linearized VlasovMaxwell equations. The analysis leads to an infinite dimension matrix dispersion equation of the form [38]

$$
\operatorname{det}\left\{D_{n, m}(\omega)\right\}=0
$$

which is valid for arbitrary normalized beam intensity $s_{b}=\hat{\omega}_{p b}^{2} / 2 \omega_{f}^{2}$ and temperature anisotropy $T_{\| b} / T_{\perp b}$. Here, the integers $n$ and $m$ label the elements of the matrix. A detailed numerical analysis [38] of the matrix dispersion relation shows that in the limit $T_{\| b} / T_{\perp b} \rightarrow 0$ the maximum growth rate of the Weibel instability asymptotes at the relatively small value

$$
(\operatorname{Im} \omega)_{\max }=0.43 \hat{\omega}_{p b} \frac{v_{\perp b}^{\mathrm{th}}}{c}
$$

for perturbations with short axial wavelength corresponding to $k_{z}^{2} r_{b}^{2} \gg 1$. Here, $v_{\perp b}^{\text {th }}=\left(2 T_{\perp b} / m_{b}\right)^{1 / 2}$ is the transverse thermal speed, and $\hat{\omega}_{p b}=\left(4 \pi \hat{n}_{b} e_{b}^{2} / m_{b}\right)^{1 / 2}$ is the on-axis $(r=0)$ plasma frequency in the beam frame. Finally, removing the restriction $T_{\| b} / T_{\perp b}=0$, a detailed numerical analysis [38] of the matrix dispersion relation in Eq. (3) shows that the Weibel instability in a onecomponent beam is completely stabilized by longitudinal thermal effects whenever $T_{\| b}$ exceeds the small threshold value $T_{\| b}^{\text {th }}$ given approximately by

$$
\frac{T_{\| b}^{\mathrm{th}}}{T_{\perp b}}=0.1 \frac{r_{b}^{2} \hat{\omega}_{p b}^{2}}{c^{2}} .
$$

To summarize, because $\hat{\omega}_{p b}^{2} r_{b}^{2} / c^{2} \ll 1$ for the beam parameters of interest for heavy ion fusion, the Weibel instability stabilizes at extremely small values of $T_{\| b}^{\text {th }} / T_{\perp b}$ [Eq. (5)]. Furthermore, in the regime where the Weibel instability does exist, the characteristic growth rate is much smaller than the growth rate of the Harris-type instability described in Sec. II A.

\section{ELECTRON-ION TWO-STREAM (ELECTRON CLOUD) INSTA BILITY IN INTENSE ION BEAMS}

In many practical accelerator applications, an unwanted charge component is present in the beam accelerator or transport lines. For example, a background population of electrons can result when energetic beam ions strike the chamber wall or ionize background gas atoms. When a second charge component is present, it has been recognized for many years, both in theoretical studies and in experimental observations [46-48,50$60]$, that the relative streaming motion of the highintensity beam particles through the background charge species provides the free energy to drive the classical twostream instability, appropriately modified to include the effects of dc space charge, relativistic kinematics, presence of a conducting wall, etc. For electrons interacting with a proton beam, as in the Proton Storage Ring (PSR), this instability is usually referred to as the electronproton $(e-p)$ instability [50,51], although a similar instability also exists for other ion species, including (for example) electron-ion interactions in electron storage rings.

We have carried out detailed theoretical investigations [52-60] of the two-stream instability for an intense ion beam propagating through a partially neutralizing electron background. These investigations have been both analytical and numerical, making use of the nonlinear perturbative particle simulation code BEST. To illustrate the qualitative features of the instability we first consider perturbations about the choice of KapchinskijVladimirskij (KV) distribution functions that have flattop density profiles [6-8]. In the laboratory frame, the equilibrium distribution functions are expressed as [5254]

$$
f_{j}^{0}(r, \mathbf{p})=\frac{\hat{n}_{j}}{2 \pi \gamma_{j} m_{j}} \delta\left(H_{\perp j}-\hat{T}_{\perp j}\right) G_{j}\left(p_{z}\right) .
$$

Here, $\quad \int d p_{z} G_{j}\left(p_{z}\right)=1, \quad H_{\perp b}=\left(p_{r}^{2}+p_{\theta}^{2}\right) / 2 \gamma_{b} m_{b}+$ $\gamma_{b} m_{b} \omega_{f}^{2} r^{2} / 2+e_{b}\left[\phi^{0}(r)-\beta_{b} A_{z}^{0}(r)\right]$ is the transverse Hamiltonian for the beam ions, $H_{\perp e}=\left(p_{r}^{2}+p_{\theta}^{2}\right) / 2 m_{e}-$ $e \phi^{0}(r)$ is the transverse Hamiltonian for the background electrons, $\hat{T}_{\perp j}=$ const $(j=b, e)$ are positive constants, and $\hat{n}_{b}$ and $\hat{n}_{e}$ are the constant values of beam density and electron density out to the edge radius $r_{b}$. The electrons are assumed to be axially stationary $\left(V_{e} \simeq 0\right)$ in the laboratory frame and are electrostatically confined in the transverse plane by the excess ion space charge. We denote the ion charge state by $e_{b}=+Z_{b} e$, and introduce the fractional charge neutralization $f$ defined by $f=\hat{n}_{e} / Z_{b} \hat{n}_{b}$.

Detailed stability properties have been calculated analytically for electrostatic perturbations about the choice of equilibrium distribution functions in Eq. (6) [52-54]. 
Without presenting algebraic details, assuming perturbations of the form $\delta \psi(\mathbf{x}, t)=\delta \hat{\psi}_{m}(r) \exp \left(\operatorname{im} \theta+i k_{z} z-\right.$ $i \omega t$ ), the dispersion relation has been derived for general azimuthal mode numbers $m=1,2,3, \ldots$ It is found that the strongest two-stream instability exists for the dipole mode with $m=1$. For example, in the limit of axially cold beam ions and electrons with $G_{b}\left(p_{z}\right)=\delta\left(p_{z}-\right.$ $\left.\gamma_{b} m_{b} \beta_{b} c\right)$ and $G_{e}\left(p_{z}\right)=\delta\left(p_{z}\right)$, the dipole-mode $(m=$ $1)$ dispersion relation is given by [52-54]

$$
\left[\left(\omega-k_{z} V_{b}\right)^{2}-\omega_{b}^{2}\right]\left[\omega^{2}-\omega_{e}^{2}\right]=\omega_{c}^{4},
$$

where

$$
\begin{aligned}
& \omega_{c}^{4}=\frac{1}{4} f\left(1-\frac{r_{b}^{2}}{r_{w}^{2}}\right)^{2} \frac{\gamma_{b} m_{b}}{Z_{b} m_{e}} \hat{\omega}_{p b}^{4}, \\
& \omega_{b}^{2}=\omega_{f}^{2}+\frac{1}{2} \hat{\omega}_{p b}^{2}\left(f-\frac{1}{\gamma_{b}^{2}} \frac{r_{b}^{2}}{r_{w}^{2}}\right), \\
& \omega_{e}^{2}=\frac{1}{2} \frac{\gamma_{b} m_{b}}{Z_{b} m_{e}} \hat{\omega}_{p b}^{2}\left(1-f \frac{r_{b}^{2}}{r_{w}^{2}}\right) .
\end{aligned}
$$

Here, $f=\hat{n}_{e} / Z_{b} \hat{n}_{b}$ is the fractional charge neutralization, and $\hat{\omega}_{p b}=\left(4 \pi \hat{n}_{b} Z_{b}^{2} e^{2} / \gamma_{b} m_{b}\right)^{1 / 2}$ is the relativistic plasma frequency of the beam ions.

In the absence of background electrons $(f=0$ and $\omega_{c}^{4}=0$ ), Eq. (7) gives stable sideband oscillations with frequency $\omega=k_{z} V_{b} \pm \omega_{b}$, where $\omega_{b}$ is defined in Eq. (8). For $f \neq 0$ and $\omega_{c}^{4} \neq 0$, however, the ion and electron terms on the left-hand side of Eq. (7) are coupled by the $\omega_{c}^{4}$ term on the right-hand side, leading to one unstable solution with $\operatorname{Im} \omega>0$. Indeed, it is the lower ion sideband $\left(\omega \simeq k_{z} V_{b}-\omega_{b}\right)$ that couples unstably to the electron oscillation $\left(\omega \simeq \omega_{e}\right)$ in Eq. (7) [52-54]. The dispersion relation (7) and its generalization to include axial momentum spread have been used to calculate detailed growth rate properties of the two-stream instability over a wide range of system parameters, including the normalized beam intensity $s_{b}=\hat{\omega}_{p b}^{2} / 2 \gamma_{b}^{2} \omega_{f}^{2}$, fractional charge neutralization $f=\hat{n}_{e} / Z_{b} \hat{n}_{b}$, and axial momentum spread $\Delta p_{z b} / p_{z b}$ of the beam ions. To briefly summarize the results described in Refs. [52-54], it is found that the normalized growth rate $(\operatorname{Im} \omega) / \omega_{f}$ (a) increases with increasing beam intensity $s_{b}$, (b) increases with increasing fractional charge neutralization $f$, and (c) decreases with increasing axial momentum spread $\Delta p_{z b} / p_{z b}$.

Extensive numerical simulations of the two-stream instability have also been carried out using the BEST nonlinear perturbative particle simulation code $[55,57,59,60]$. In the simulations, perturbations are about the choice of equilibrium distribution function $(j=b, e)$

$$
f_{j}^{0}(r, \mathbf{p})=\frac{\hat{n}_{j}}{2 \pi \gamma_{j} m_{j} T_{\perp j}} \exp \left(-\frac{H_{\perp j}}{T_{\perp j}}\right) G_{j}\left(p_{z}\right) .
$$

Here, $\hat{n}_{j}$ is the on-axis $(r=0)$ density, $T_{\perp_{j}}=$ const is the transverse temperature, and $G_{j}\left(p_{z}\right)$ is the longitudinal momentum distribution. For the beam ions we take
$G_{b}\left(p_{z}\right)$ to be a drifting Maxwellian centered at $p_{z}=$ $\gamma_{b} m_{b} \beta_{b} c$, and for the background electrons we take $G_{e}\left(p_{z}\right)$ to be a Maxwellian centered at $p_{z}=0$. An important feature of Eq. (9) is that the corresponding density profiles $n_{j}^{0}(r)=\int d^{3} p f_{j}^{0}(r, \mathbf{p})$ are generally bellshaped functions of $r$. For $f=\hat{n}_{e} / Z_{b} \hat{n}_{b}=0$, only in the space-charge-dominated limit, where $s_{b}=$ $\hat{\omega}_{p b}^{2} / 2 \gamma_{b}^{2} \omega_{f}^{2} \rightarrow 1$, does the ion beam density profile become flattop. Here, $\hat{\omega}_{p b}=\left(4 \pi \hat{n}_{b} Z_{b}^{2} e^{2} / \gamma_{b} m_{b}\right)^{1 / 2}$ is the (on-axis) relativistic plasma frequency. An important consequence of the bell-shaped density profile shape is that the growth rate observed in the numerical simulations $[55,57,59,60]$ are typically lower than those predicted theoretically for flattop density profiles assuming perturbations about a KV equilibrium. This is likely due to the spread in depressed betatron frequency associated with the nonuniform density profiles.

Detailed simulations of the electron-ion two-stream instability have been carried out using the BEST code $[55,57,59,60]$ for applications ranging from proton machines, such as the PSR experiment, to heavy ion fusion. Some illustrative results for heavy ion fusion are presented in Figs. 4-6. Here, we take singly charged cesium ions $\left(Z_{b}=1\right.$ and $\left.A=133\right)$ with relativistic mass factor $\gamma_{b}=1.02$. The beam intensity is taken to be near the space-charge-dominated limit $\left(s_{b} \rightarrow 1\right)$ in the absence of electrons. The on-axis fractional charge neutralization is taken to be $f=\hat{n}_{e} / \hat{n}_{b}=0.1$, and the transverse temperatures are $T_{b \perp} / \gamma_{b} m_{b} V_{b}^{2}=1.1 \times 10^{-6}$ and $T_{e \perp} / \gamma_{b} m_{b} V_{b}^{2}=2.47 \times 10^{-6}$. The corresponding ion and electron density profile are bell shaped and overlap radially. In the simulations, after small-amplitude perturbations are excited at $t=0$, the system is evolved selfconsistently for thousands of wave periods. Plotted in Fig. 4 is the time history of the beam density perturbation

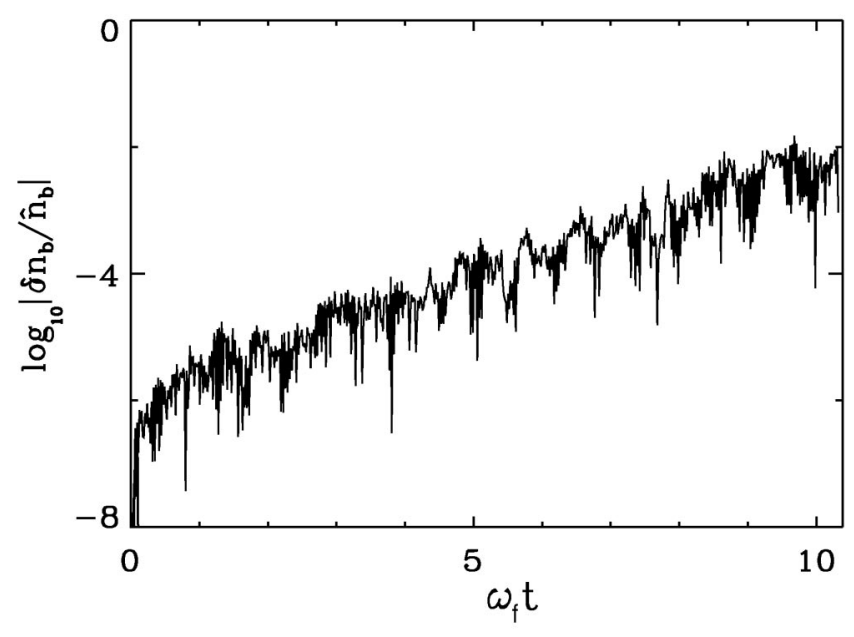

FIG. 4. Time history of perturbed density $\delta n_{b} / \hat{n}_{b}$ at a fixed spatial location. After an initial transition period, the $m=1$ dipole-mode perturbation grows exponentially. 


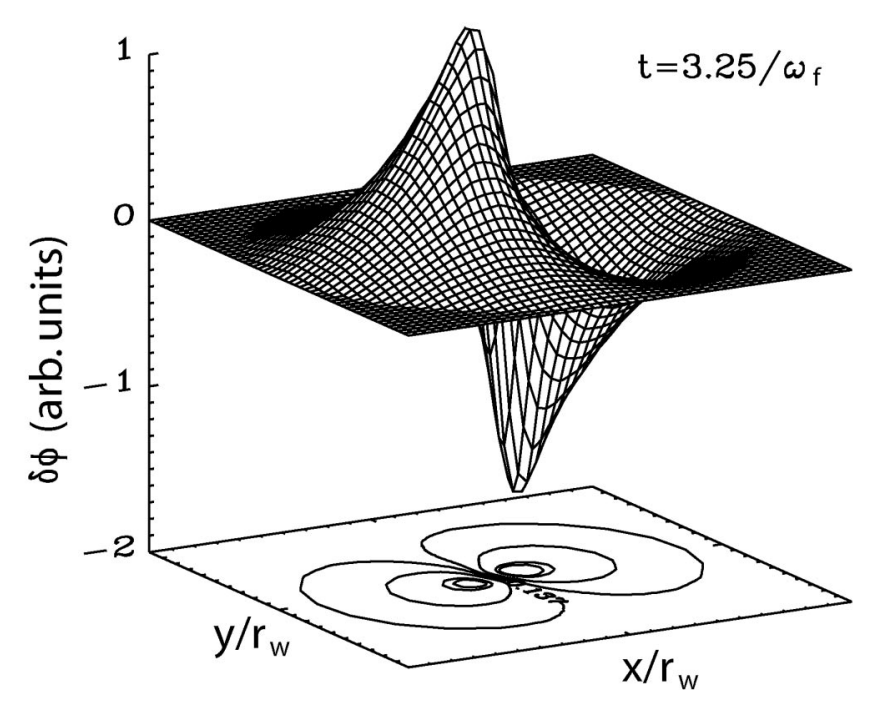

FIG. 5. The $x-y$ projection (at fixed value of $z$ ) of the perturbed electrostatic potential $\delta \phi(x, y, t)$ for the ion-electron two-stream instability growing from a small initial perturbation, shown at $\omega_{f} t=3.25$.

at one spatial location in a simulation using the linearized version of the BEST code. Evidently, after an initial transition period, the perturbation grows exponentially, which is the expected behavior of an instability during the linear growth phase. In Fig. 5, the $x-y$ projections of the perturbed potential $\delta \phi$ at a fixed longitudinal position are plotted at $t=0$ and $t=3.25 / \omega_{f}$. Clearly, $\delta \phi$ grows to a moderate amplitude by $t=3.25 / \omega_{f}$, and the $m=1$ dipole mode is the dominant unstable mode, for which the growth rate is measured to be $\operatorname{Im} \omega=0.78 \omega_{f}$. The real eigenfrequency of the mode is $\operatorname{Re} \omega=480 \omega_{f}$, and the normalized wavelength at maximum growth is $k_{z} V_{b} / \omega_{f}=480.4$.

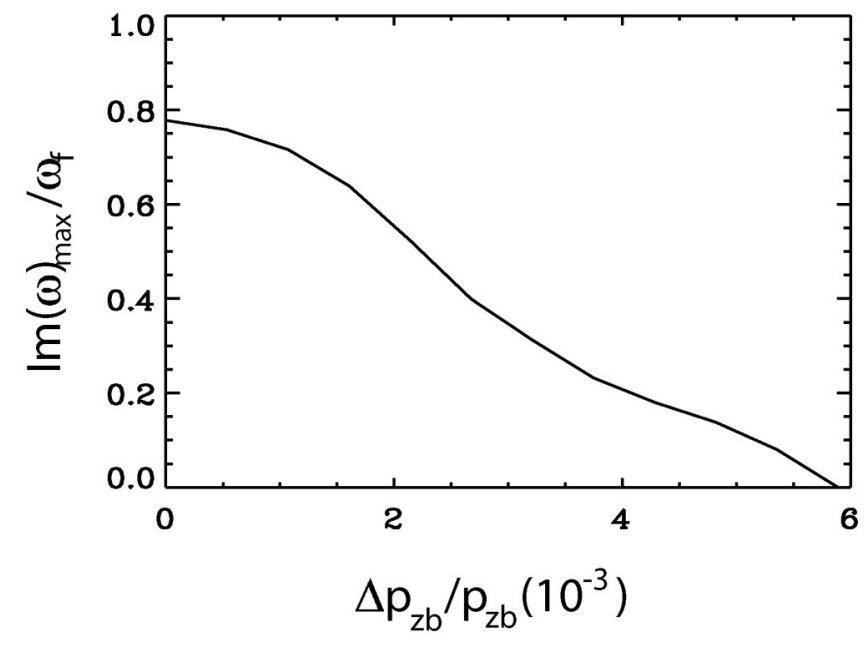

FIG. 6. The maximum linear growth rate $(\operatorname{Im} \omega)_{\max }$ of the ion-electron two-stream instability decreases as the longitudinal momentum spread of the beam ions increases.
In Figs. 4 and 5, we have assumed initially cold beam ions in the longitudinal direction $\left(\Delta p_{z b} / p_{z b}=0\right)$ to maximize the growth rate of the instability. Here, $p_{z b}=$ $\gamma_{b} m_{b} V_{b}$. In general, when the longitudinal momentum spread of the beam ions is finite, Landau damping by parallel ion kinetic effects provides a mechanism that reduces the growth rate. Shown in Fig. 6 is a plot of the maximum linear growth rate $(\operatorname{Im} \omega)_{\max }$ versus the normalized initial axial momentum spread $\Delta p_{z b} / p_{z b}$ obtained in the numerical simulations. As is evident from Fig. 6, the growth rate decreases dramatically as $\Delta p_{z b} / p_{z b}$ is increased. When $\Delta p_{z b} / p_{z b}$ is high enough, about $0.58 \%$ for the case in Fig. 6 , the mode is completely stabilized by longitudinal Landau damping effects by the beam ions. This result agrees qualitatively with theoretical predictions.

\section{INTENSE ION BEAM INTERACTION WITH BACKGROUND PLASMA}

In previous sections we have investigated anisotropydriven collective instabilities in one-component ion beams (Sec. II) and the dipole-mode two-stream instability driven by the beam ions interacting with background electrons that provide partial charge neutralization (Sec. III). In this section, we discuss several collective instabilities that can occur when an intense ion beam $(j=b)$ propagates through a chargeneutralizing background plasma $(j=e, i)$ in the plasma plug or neutralized drift compression region, and in the target chamber. Particular emphasis is placed on the resistive hose instability [61-70], and the multispecies electrostatic two-stream and electromagnetic Weibel instabilities [71-73]. Here, the Weibel instability is associated with the anisotropy associated with the relative directed kinetic energy of the beam-plasma components. Throughout Sec. IV, it is assumed that under quasi-steadystate conditions the background plasma provides a charge-neutralizing background [74-77] with $\sum_{j=b, e, i} n_{j}^{0}(r) e_{j}=0$. It is further assumed that the beamplasma interaction takes place in a region where there is no applied focusing field $\left(\omega_{f}=0\right)$, and that a perfectly conducting cylindrical wall is located at radius $r=r_{w}$.

\section{A. Resistive hose instability}

The resistive hose instability [61-67] has received considerable attention for intense electron-beam propagation through the atmosphere or background plasma. In this section, we briefly summarize recent theoretical results [67] obtained for the case of an intense ion beam propagating through a charge-neutralizing background plasma, including the important influence of electron collisions on reducing the growth rate of the resistive hose instability. For simplicity, we consider dipolemode perturbations about an intense ion beam with 
Kapchinskij-Vladimirskij distribution [Eq. (6)] and flattop density profile

$$
n_{b}^{0}(r)= \begin{cases}\hat{n}_{b}=\mathrm{const}, & 0 \leq r<r_{b} \\ 0, & r_{b}<r \leq r_{w}\end{cases}
$$

It is also assumed in the present analysis that the beam ions are cold in the longitudinal direction with $G_{b}\left(p_{z}\right)=$ $\delta\left(p_{z}-\gamma_{b} m_{b} \beta_{b} c\right)$. While providing complete charge neutralization, the background plasma is allowed to carry an axial return current $J_{z p}^{0}=\sum_{j=e, i} \hat{n}_{j} e_{j} \beta_{j} c=$ $-f_{m}\left(\hat{n}_{b} e_{b} \beta_{b} c\right)$, where $f_{m}=$ const is the fractional current neutralization, and $V_{z j}=\beta_{j} c=$ const is the average axial velocity of the background plasma components $(j=$ $e, i)$. The instability is electromagnetic and is caused by the interaction between the transversely displaced beam current $J_{b z}$ and the induced transverse magnetic field $\delta \mathbf{B}^{T}=\delta B_{r} \hat{\mathbf{e}}_{r}+\delta B_{\theta} \hat{\mathbf{e}}_{\theta}$. Therefore, the main component of perturbed current is in the $z$ direction and the perturbed electric field is also in the $z$ direction. Such a field polarization can be represented with one component of the vector potential $A_{z}$. Therefore, the transverse electromagnetic field perturbations are assumed to have components $\delta \mathbf{E}^{T}=\delta E_{z} \hat{\mathbf{e}}_{z} \quad$ and $\delta \mathbf{B}^{T}=\delta B_{r} \hat{\mathbf{e}}_{r}+\delta B_{\theta} \hat{\mathbf{e}}_{\theta}$, where $\delta \mathbf{E}^{T}=-c^{-1}(\partial / \partial t) \delta A_{z} \hat{\mathbf{e}}_{z}$ and $\delta \mathbf{B}^{T}=\nabla \times \delta A_{z} \hat{\mathbf{e}}_{z}$. Introducing the time variable $\tau=t-z / V_{b}$ measured from the head of the beam pulse (passing $z=0$ at $t=$ $0)$, the perturbed vector potential can be expressed as

$$
\delta A_{z}(\mathbf{x}, t)=\delta \hat{A}_{z}(r) \exp \left[i\left(\theta-\Omega z / V_{b}-\omega \tau\right)\right]
$$

for dipole-mode perturbations with azimuthal mode number $m=1$. Here, $\Omega=\omega-k_{z} V_{b}$ is the Dopplershifted frequency in the beam frame. If, for example, the beam experiences a transverse perturbation with real frequency $\omega$ as the beam pulse enters the plasma, then it follows from Eq. (11) that $(\operatorname{Im} \Omega) / V_{b}$ represents the spatial growth rate of the instability along the beam pulse. Finally, the perturbed plasma current is determined from $\delta J_{z p}=\sigma \delta E_{z}=(i \omega \sigma / c) \delta A_{z}$, where the plasma conductivity $\sigma$ is given by the simple model [67]

$$
\sigma=\frac{1}{1-i \omega / \nu_{c}} \begin{cases}\sigma_{p}, & 0 \leq r<r_{b}, \\ \sigma_{1}, & r_{b}<r \leq r_{w} .\end{cases}
$$

Here, $\sigma_{p}$ and $\sigma_{1}$ are the dc plasma conductivities in the two regions, and $\nu_{c}$ is the electron collision frequency. The frequency $\omega$ is typically of order the transverse betatron frequency, which is determined by the beam density. On the other hand, the electron collision frequency $\nu_{c}$ in Eq. (12) is determined by plasma properties. In this context, the parameter $\omega / \nu_{c}$ in Eq. (12) can be larger or smaller than unity, depending on the system parameters.

Making use of the assumptions enumerated in the previous paragraph, the linearized Vlasov-Maxwell equations for $\delta A_{z}(\mathbf{x}, t)$ and $\delta f_{b}(\mathbf{x}, \mathbf{p}, t)$ can be used to calculate the perturbed axial current $\delta J_{z b}=$ $e_{b} \int d^{3} p v_{z} \delta f_{b}$ carried by the beam ions, and derive a transcendental dispersion relation that determines the complex frequency $\Omega$ in terms of $\omega, \sigma_{p}, \sigma_{1}, \hat{n}_{b}$, etc. We consider here the particular case where $|\omega| \sigma_{1} \ll$ $c^{2} / 4 \pi r_{b}^{2}$, which assures that magnetic diffusion through the weakly conducting region $r_{b}<r \leq r_{w}$ is fast compared with the time scale $\omega^{-1}$. Without presenting algebraic details [67], this leads to the dispersion relation

$$
\frac{\hat{\omega}_{p b}^{2} \beta_{b}^{2}}{\Omega^{2}-\omega_{\beta}^{2}}=-\kappa_{p} r_{b} \frac{J_{1}^{\prime}\left(\kappa_{p} r_{b}\right)}{J_{1}\left(\kappa_{p} r_{b}\right)}-\frac{r_{w}^{2}+r_{b}^{2}}{r_{w}^{2}-r_{b}^{2}},
$$

where $\hat{\omega}_{p b}=\left(4 \pi \hat{n}_{b} e_{b}^{2} / \gamma_{b} m_{b}\right)^{1 / 2}$ is the relativistic plasma frequency of the beam ions, $J_{1}(x)$ is the Bessel function of the first kind of order unity, and $\omega_{\beta}^{2}$ and $\kappa_{p}^{2}(\omega)$ are defined by

$$
\begin{gathered}
\omega_{\beta}^{2}=\frac{1}{2} \hat{\omega}_{p b}^{2} \beta_{b}^{2}\left(1-f_{m}\right), \\
\kappa_{p}^{2}(\omega) r_{b}^{2}=\frac{8 i \omega \tau_{d}}{\left(1-i \omega / \nu_{c}\right)} .
\end{gathered}
$$

Here, $\tau_{d} \equiv \pi \sigma_{p} r_{b}^{2} / 2 c^{2}$ is the magnetic decay time for the perturbed current, and $\omega_{\beta}$ is the betatron oscillation frequency for transverse motion of the beam particles in the equilibrium azimuthal self-magnetic field $B_{\theta}^{0}(r)$ associated with the net axial current. If we further assume $\left|\kappa_{p} r_{b}\right|<1$, then Eq. (13) reduces to leading order to

$$
\frac{\hat{\omega}_{p b}^{2} \beta_{b}^{2} / 2}{\Omega^{2}-\omega_{\beta}^{2}}=i \frac{\omega \tau_{d}}{1-i \omega / \nu_{c}}-g,
$$

where $g=\left(1-r_{b}^{2} / r_{w}^{2}\right)^{-1}$ is a geometric factor. Equation (16) can be used to investigate detailed stability properties over a wide range of system parameters. As one simple limiting case, for $|\omega| \tau_{d} \rightarrow 0$, Eq. (16) reduces to

$$
\Omega^{2}=\frac{\left[\left(1-f_{m}\right) g-1\right]}{\left(1-f_{m}\right) g} \omega_{\beta}^{2} .
$$

Note that Eq. (17) yields the familiar return-current instability $\left(\Omega^{2}<0\right)$ whenever $f_{m}$ exceeds the critical value

$$
f_{m}>f_{c} \equiv \frac{g-1}{g}=\frac{r_{b}^{2}}{r_{w}^{2}} .
$$

Equation (16) can also be used to investigate detailed stability properties that depend on the conductivity $\sigma_{p}$ of the plasma channel and the electron collision frequency $\nu_{c}$. Typical results are illustrated in Fig. 7 for the case where $\omega \tau_{d}=0.075$ and $f_{m}=0$. In Fig. 7, the normalized growth rate $\operatorname{Im} \Omega / \omega_{\beta}$ is plotted versus the geometrical factor $g=\left(1-r_{b}^{2} / r_{w}^{2}\right)^{-1}$ for $g$ ranging from 1 to 2 , and several values of the parameter $\omega / \nu_{c}$. Note that $g=1$ corresponds to $r_{w}^{2} / r_{b}^{2} \rightarrow \infty$, whereas $g=2$ corresponds to a nearby conducting wall with $r_{b} \simeq 0.7 r_{w}$. As expected, the proximity of a conducting wall greatly reduces the 


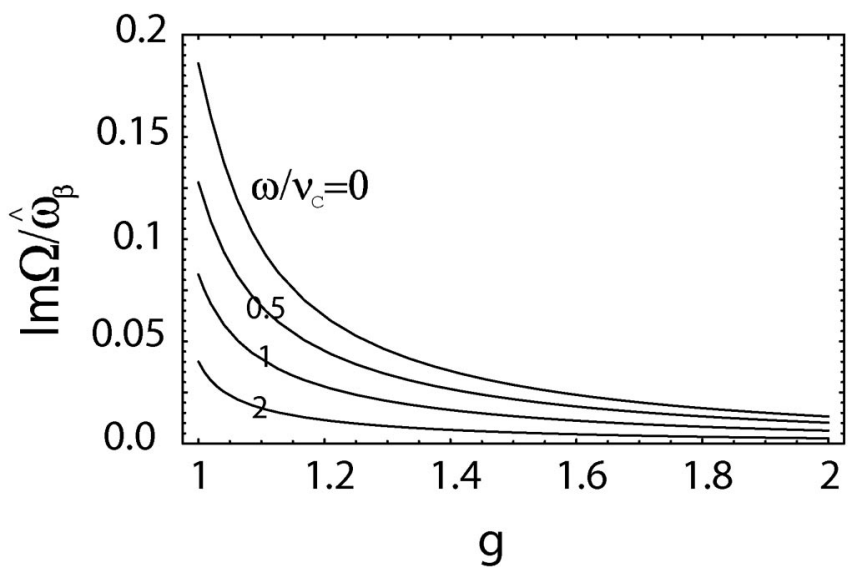

FIG. 7. Plots of the normalized growth rate $\operatorname{Im} \Omega / \omega_{\beta}$ versus the geometrical factor $g$ obtained from Eq. (16) for several values of the frequency ratio $\omega / \nu_{c}$, and $\omega \tau_{d}=0.075$ and $f_{m}=0$.

growth rate of the resistive hose instability. Furthermore, the normalized growth rate $\operatorname{Im} \Omega / \omega_{\beta}$ decreases for increasing values of $\omega / \nu_{c}$, although the normalized oscillation frequency is relatively insensitive to the value of $\omega / \nu_{c}$ [67]. Note also from Fig. 7 that the growth rate of the resistive hose instability can be substantial, even when $\omega^{2} \tau_{d}^{2} \ll 1$. For example, from Fig. 1 , for $g=1$ and $\omega / \nu_{c}=0.5$, we obtain $\operatorname{Im} \Omega=0.125 \omega_{\beta}$.

As an illustrative example characteristic of heavy ion fusion applications, we consider a $1 \mathrm{kA}$ cesium ion beam, where the beam ions are singly charged with $Z_{b}=1$, and the average kinetic energy is $\left(\gamma_{b}-1\right) m_{b} c^{2}=2.5 \mathrm{GeV}$ corresponding to $\beta_{b}=0.2$. Assuming that the beam radius is $r_{b}=1 \mathrm{~cm}$, the beam density is calculated to be $n_{b}=3.4 \times 10^{11} \mathrm{~cm}^{-3}$. The corresponding betatron frequency calculated from Eq. (16) is $\omega_{\beta}=9.2 \times$ $10^{6} \mathrm{~s}^{-1}$, assuming zero return current $\left(f_{m}=0\right)$. The electron collision frequency for Coulomb collisions is given by $\nu_{c}=2.9 \times 10^{-6} n_{e} 1 n \Lambda T_{e}^{-3 / 2}$, where the typical value of the Coulomb logarithm is about $1 n \Lambda=10$. Assuming the electron temperature is about $T_{e}=1 \mathrm{eV}$ and taking $n_{e}=n_{b}=10^{12} \mathrm{~cm}^{-3}$, the conductivity of the background plasma for this choice of parameters is estimated to be $\sigma=3 \times 10^{12} \mathrm{~s}^{-1}$. Therefore, the magnetic decay time is calculated to be $\tau_{d}=5 \times 10^{-9} \mathrm{~s}$. Assuming the characteristic value of real frequency is $\omega \simeq \omega_{\beta}$ at $z=0$, we obtain $\omega \tau_{d}=7.5 \times 10^{-2}$, which is much less than unity. Substituting into Eq. (16), the instability growth rate is $\operatorname{Im} \Omega=\Omega_{i}=0.13 \omega_{\beta}$ for $g \rightarrow 1$, and the corresponding real oscillation frequency is $\operatorname{Re} \Omega=-0.22 \omega_{\beta}$. Note that the growth rate of the resistive hose instability can be a substantial fraction of the betatron frequency of the beam particles for this choice of system parameters.

In summary, it is important to recognize that there are several mechanisms for reducing the growth rate of the resistive hose instability. Growth rate reduction mechanisms include (a) increasing the characteristic value of $|\omega| / \nu_{c}$; (b) proximity of a conducting wall (increasing values of $r_{b} / r_{w}$ ); and (c) decreasing the value of fractional current neutralization $f_{m}$. In addition, rounded beam density profiles tend to give lower growth rates than the flattop density profile in Eq. (10) [61].

In concluding this section, it is important to recognize that the resistive hose instability may play an important role for ion beam propagation through a dense plasma channel when the electrons are relatively cold and the resistivity is correspondingly high. On the other hand, for charge-neutralized ballistic transport, when the plasma density is lower and the electrons have higher temperatures, the resistive hose instability is likely to play a less important role because of the lower resistivity.

\section{B. Multispecies Weibel instability}

The electromagnetic Weibel instability [37-42] was shown in Sec. II B to be relatively ineffective in onecomponent charged particle beams. The situation can be quite different, however, when an intense beam propagates through background plasma [39-42,72,73]. In this case, the large energy anisotropy associated with the directed kinetic energy of the beam particles relative to the background plasma can provide significant free energy to drive the transverse electromagnetic Weibel instability, and cause filamentation in the plane perpendicular to beam propagation. In this section, we summarize the results of a recent calculation [73] based on a macroscopic cold-fluid model in which an intense ion beam $(j=b)$ propagates through a background plasma $(j=e, i)$. The background plasma is assumed to provide complete charge and current neutralization with

$$
\sum_{j=b, e, i} n_{j}^{0}(r) e_{j}=0 \quad \text { and } \quad \sum_{j=b, e, i} n_{j}^{0}(r) e_{j} \beta_{j} c=0 .
$$

Here, $V_{z j}=\beta_{j} c$ is the average axial velocity (assumed constant) of species $j(j=b, e, i)$, and $\gamma_{j}=\left(1-\beta_{j}^{2}\right)^{-1 / 2}$ is the relativistic mass factor. In Eq. (19), current neutralization has been assumed since this case tends to give the largest growth rate for the multispecies Weibel instability [83]. That is, a finite azimuthal self-magnetic field $B_{\theta}^{0}(r) \neq 0$ tends to reduce the growth rate of the Weibel instability $[41,83]$. Furthermore, the present analysis assumes axisymmetric flute perturbations with $\partial / \partial \theta=0$ and $\partial / \partial z=0$, and electromagnetic field perturbations with components $\delta \mathbf{E}=\delta E_{r} \hat{\mathbf{e}}_{r}+\delta E_{z} \hat{\mathbf{e}}_{z}$ and $\delta \mathbf{B}=$ $\delta B_{\theta} \hat{\mathbf{e}}_{\theta}$. Note that the field perturbations assumed here have mixed polarization with both a longitudinal component $\left(\delta E_{r} \neq 0\right)$ and the transverse electromagnetic components $\left(\delta B_{\theta} \neq 0\right.$ and $\left.\delta E_{z} \neq 0\right)$. Finally, similar to Sec. IVA, it is assumed that the beam-plasma interactions take place in a region where there is no applied 
focusing field $\left(\omega_{f}=0\right)$, and there is a perfectly conducting cylindrical wall located at radius $r=r_{w}$.

Within the context of the assumptions enumerated in the previous paragraph, we express $\delta E_{z}(r, t)=\delta \hat{E}_{z}(r) \times$ $\exp (-i \omega t)$, where $\operatorname{Im} \omega>0$ corresponds to instability (temporal growth). Making use of a cold-fluid model that neglects pressure perturbations, this leads to the eigenvalue equation [73]

$$
\begin{aligned}
\frac{1}{r} \frac{\partial}{\partial r}\left[r \left(1+\sum_{j=b, e, i} \frac{\beta_{j}^{2} \omega_{p j}^{2}(r)}{\omega^{2}}\right.\right. & \\
& \left.\left.+\frac{\left[\sum_{j=b, e, i} \beta_{j} \omega_{p j}^{2}(r)\right]^{2}}{\omega^{2}\left[\omega^{2}-\sum_{j=b, e, i} \omega_{p j}^{2}(r)\right]}\right) \frac{\partial}{\partial r} \delta \hat{E}_{z}\right] \\
& +\left(\frac{\omega^{2}}{c^{2}}-\sum_{j=b, e, i} \frac{\omega_{p j}^{2}(r)}{\gamma_{j}^{2} c^{2}}\right) \delta \hat{E}_{z}=0
\end{aligned}
$$

where $\quad \omega_{p j}(r)=\left[4 \pi n_{j}^{0}(r) e_{j}^{2} / \gamma_{j} m_{j}\right]^{1 / 2}$ and $\gamma_{j}=\left(1-\beta_{j}^{2}\right)^{-1 / 2}$.

Equation (20) is the desired eigenvalue equation for axisymmetric, ordinary-mode electromagnetic perturbations, with the terms proportional to $\sum_{j=b, e, i} \beta_{j}^{2} \omega_{p j}^{2}(r)$ and $\sum_{j=b, e, i} \beta_{j} \omega_{p j}^{2}(r) \neq 0$ providing the free energy to drive the Weibel instability. Equation (20) can be integrated numerically to determine the eigenvalue $\omega^{2}$ and eigenfunction $\delta E_{z}(r)$ for a wide range of beam-plasma density profiles $n_{j}^{0}(r)$. Analytical solutions are also tractable for the case of flattop (step function) density profiles. As a general remark, when $\sum_{j=b, e, i} \beta_{j}^{2} \omega_{p j}^{2}(r) \neq 0$ and $\sum_{j=b, e, i} \beta_{j} \omega_{p j}^{2}(r) \neq 0$, Eq. (20) supports both stable fastwave solutions $\left(\operatorname{Im} \omega=0,\left|\omega / c k_{\perp}\right|>1\right)$ and unstable slow-wave solutions $\left(\operatorname{Im} \omega>0,\left|\omega / c k_{\perp}\right|<1\right)$. Here, $\left|k_{\perp}\right| \sim|\partial / \partial r|$ is the characteristic radial wave number of the perturbation. Equation (20) also supports plasma oscillation solutions with predominantly longitudinal polarization associated with the factor proportional to $\left[\omega^{2}-\sum_{j=b, e, i} \omega_{p j}^{2}(r)\right]^{-1}$.

As an example that is analytically tractable, we consider the case where the density profiles are uniform both inside and outside the beam with

$$
n_{j}^{0}(r)=\hat{n}_{j}^{i}=\mathrm{const}, \quad j=b, e, i,
$$

for $0 \leq r<r_{b}$, and

$$
n_{j}^{0}(r)=\hat{n}_{j}^{o}=\mathrm{const}, \quad j=e, i,
$$

for $r_{b}<r \leq r_{w}$. Here, the superscript " $i$ " (" $o$ ") denotes inside (outside) the beam, and $\hat{n}_{b}^{o}=0$ is assumed. Consistent with Eq. (19), $\quad \sum_{j=b, e, i} \hat{n}_{j}^{i} e_{j}=0=$ $\sum_{j=b, e, i} \hat{n}_{j}^{i} \beta_{j} e_{j}$ and $\sum_{j=e, i} \hat{n}_{j}^{o} e_{j}=0=\sum_{j=e, i} \hat{n}_{j}^{o} \beta_{j} e_{j}$ are assumed. We also take $\beta_{j}=0(j=e, i)$ in the region outside the beam $\left(r_{b}<r \leq r_{w}\right)$. Analysis of the eigen- value equation (20) is able to treat the three cases: (a) beam-plasma-filled waveguide $\left(r_{b}=r_{w}\right)$; (b) vacuum region outside the beam $\left(r_{b}<r_{w}\right.$ and $\hat{n}_{j}^{o}=$ $0, j=e, i)$; and (c) plasma outside the beam $\left(r_{b}<r_{w}\right.$ and $\left.\hat{n}_{j}^{o} \neq 0, j=e, i\right)$. Referring to Eq. (20), it is convenient to introduce the constant coefficients

$$
\begin{aligned}
T_{i}^{2}(\omega)= & {\left[\frac{\omega^{2}}{c^{2}}-\sum_{j=b, e, i} \frac{\hat{\omega}_{p j}^{i 2}}{\gamma_{j}^{2} c^{2}}\right]\left[1+\frac{1}{\omega^{2}} \sum_{j=b, e, i} \beta_{j}^{2} \hat{\omega}_{p j}^{i 2}\right.} \\
& \left.+\frac{\left(\sum_{j=b, e, i} \beta_{j} \hat{\omega}_{p j}^{i 2}\right)^{2}}{\omega^{2}\left[\omega^{2}-\sum_{j=b, e, i} \hat{\omega}_{p j}^{i 2}\right]}\right]^{-1}
\end{aligned}
$$

for $0 \leq r<r_{b}$, and

$$
T_{o}^{2}(\omega)=-\left[\frac{\omega^{2}}{c^{2}}-\sum_{j=e, i} \frac{\hat{\omega}_{p j}^{02}}{c^{2}}\right]
$$

for $r_{b}<r \leq r_{w}$, where $\hat{\omega}_{p j}^{i 2}=4 \pi \hat{n}_{j}^{i} e_{j}^{2} / \gamma_{j} m_{j}, j=b, e, i$ and $\hat{\omega}_{p j}^{o 2}=4 \pi \hat{n}_{j}^{o} e_{j}^{2} / m_{j}, j=e, i$. Solving Eq. (20) for the choice of density profiles in Eqs. (21) and (22) and enforcing $\delta \hat{E}_{z}\left(r=r_{w}\right)=0$, some straightforward algebraic manipulation gives the transcendental dispersion relation [73]

$$
\begin{gathered}
\left(1+\frac{1}{\omega^{2}} \sum_{j=b, e, i} \beta_{j}^{2} \hat{\omega}_{p j}^{i 2}+\frac{\left(\sum_{j=b, e, i} \beta_{j} \hat{\omega}_{p j}^{i 2}\right)^{2}}{\omega^{2}\left[\omega^{2}-\sum_{j=b, e, i} \hat{\omega}_{p j}^{i 2}\right]}\right) T_{i} r_{b} \frac{J_{0}^{\prime}\left(T_{i} r_{b}\right)}{J_{0}\left(T_{i} r_{b}\right)} \\
=T_{o} r_{b} \frac{K_{0}\left(T_{o} r_{w}\right) I_{0}^{\prime}\left(T_{o} r_{b}\right)-K_{0}^{\prime}\left(T_{o} r_{b}\right) I_{0}\left(T_{o} r_{w}\right)}{K_{0}\left(T_{o} r_{w}\right) I_{0}\left(T_{o} r_{b}\right)-K_{0}\left(T_{o} r_{b}\right) I_{0}\left(T_{o} r_{w}\right)}
\end{gathered}
$$

Here, $T_{i}(\omega)$ and $T_{o}(\omega)$ are defined in Eqs. (23) and (24), and $I_{0}^{\prime}(x)=(d / d x) I_{0}(x), J_{0}^{\prime}(x)=(d / d x) J_{0}(x)$, etc., where $I_{0}(x)$ and $K_{0}(x)$ are modified Bessel functions, and $J_{0}(x)$ is the Bessel function of the first kind of order zero.

The dispersion relation (25) has been solved numerically [73] for the complex oscillation frequency $\omega$ for a wide range of system parameters corresponding to (a) plasma-filled waveguide $\left(r_{b}=r_{w}\right)$; (b) plasma outside the beam-plasma channel $\left(\hat{n}_{j}^{o} \neq 0, j=e, i\right.$, and $\left.r_{b}<r_{w}\right)$; and (c) no plasma outside the beam-plasma channel $\left(\hat{n}_{j}^{o}=\right.$ $0, j=e, i$, and $\left.r_{b}<r_{w}\right)$. As an illustrative example, we consider here the case where $r_{b}<r_{w}$ and there is no plasma outside the beam-plasma channel, i.e., $\hat{n}_{j}^{o}=0$ and $T_{o}^{2}(\omega)=-\omega^{2} / c^{2}$ in Eqs. (24) and (25). Inside the beam-plasma channel $\left(0 \leq r<r_{b}\right)$, it is important to recognize the relative size of the various beam-plasma species contributing to the instability drive terms $\sum_{j=b, e, i} \beta_{j}^{2} \hat{\omega}_{p j}^{i 2}$ and $\sum_{j=b, e, i} \beta_{j} \hat{\omega}_{p j}^{i 2}$ in the definition of $T_{i}^{2}(\omega)$ in Eq. (23). Assuming a positively charged ion beam $(j=b)$ propagating through background plasma electrons and ions $(j=e, i)$, the charge states are denoted by $e_{b}=+Z_{b} e, e_{e}=-e$, and $e_{i}=+Z_{i} e$, and the plasma electrons are assumed to carry the neutralizing current 

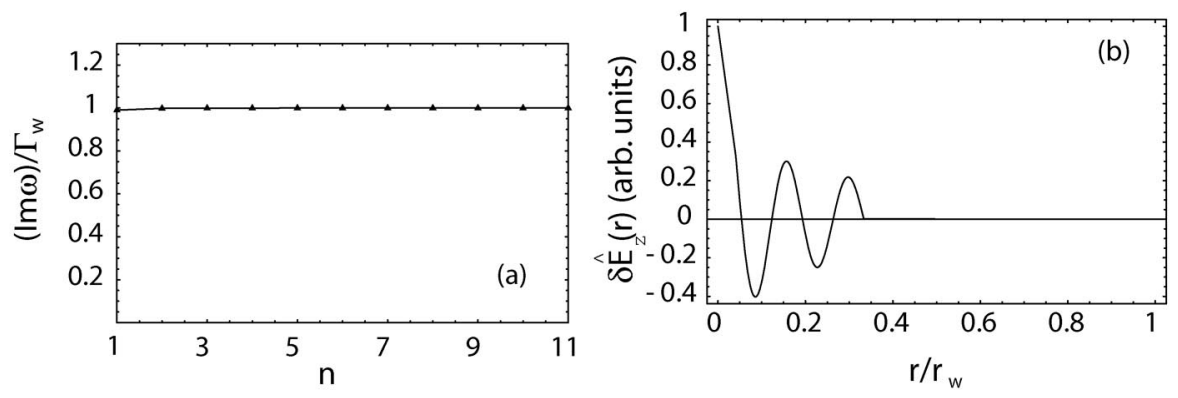

FIG. 8. Plots of (a) Weibel instability growth rate $(\operatorname{Im} \omega) / \Gamma_{W}$ versus mode number $n$, and (b) eigenfunction $\delta \hat{E}_{z}(r)$ versus $r / r_{w}$ for $n=5$ obtained from Eqs. (20) and (25). System parameters are $r_{b}=r_{w} / 3, \beta_{b}=0.2, \Omega_{p}^{i} r_{b} / c=1 / 3$, and $\Omega_{p}^{0}=0$.

$\left(\beta_{e} \neq 0\right)$, whereas the plasma ions are taken to be stationary $\left(\beta_{i}=0\right)$. The conditions for charge neutralization, $\sum_{j=b, e, i} \hat{n}_{j}^{i} e_{j}=0$, and current neutralization, $\sum_{j=b, e, i} \hat{n}_{j}^{i} e_{j} \beta_{j}=0$, then give

$$
\hat{n}_{e}^{i}=Z_{b} \hat{n}_{b}^{i}+Z_{i} \hat{n}_{i}^{i}, \quad \beta_{e}=\frac{\beta_{b} Z_{b} \hat{n}_{b}^{i}}{Z_{b} \hat{n}_{b}^{i}+Z_{i} \hat{n}_{i}^{i}}
$$

Except for the case of a very tenuous beam $\left(Z_{b} \hat{n}_{b}^{i} \ll\right.$ $Z_{i} \hat{n}_{i}^{i}$ ), note from Eq. (26) that $\beta_{e}$ can be a substantial fraction of $\beta_{b}$.

In the analysis of the dispersion relation (25), it is useful to define

$$
\begin{aligned}
& \Omega_{p}^{i 2} \equiv \sum_{j=b, e, i} \hat{\omega}_{p j}^{i 2}, \quad \Omega_{p}^{o 2} \equiv \sum_{j=e, i} \hat{\omega}_{p j}^{o 2}, \\
&\left\langle\beta^{2}\right\rangle \equiv \frac{\sum_{j=b, e, i} \beta_{j}^{2} \hat{\omega}_{p j}^{i 2}}{\sum_{j=b, e, i} \hat{\omega}_{p j}^{i 2}}, \quad\langle\beta\rangle \equiv \frac{\sum_{j=b, e, i} \beta_{j} \hat{\omega}_{p j}^{i 2}}{\sum_{j=b, e, i} \hat{\omega}_{p j}^{i 2}},
\end{aligned}
$$

where $\hat{\omega}_{p j}^{i 2}=4 \pi \hat{n}_{j}^{i} e_{j}^{2} / \gamma_{j} m_{j}, \quad \gamma_{j}=\left(1-\beta_{j}^{2}\right)^{-1 / 2} \quad$ and $\hat{\omega}_{p j}^{o 2}=4 \pi \hat{n}_{j}^{o} e_{j}^{2} / m_{j}$. Note from Eq. (27) that $\sum_{j=b, e, i} \hat{\omega}_{p j}^{i 2} / \gamma_{j}^{2} \equiv \Omega_{p}^{i 2}-\left\langle\beta^{2}\right\rangle \Omega_{p}^{i 2}$. For the case where there is a vacuum region outside the beam-plasma channel, i.e., $r_{b}<r_{w}$ and $\hat{n}_{j}^{o}=0, j=e, i$, then $T_{o}^{2}(\omega)=$ $-\omega^{2} / c^{2}$ and $\Omega_{p}^{o 2}=0$ follow from Eqs. (24) and (27), and the full transcendental dispersion relation (25) must be solved numerically. Both stable (fast wave and plasma oscillation) and unstable (Weibel-like) solutions are found. Careful examination of Eq. (25) for shortwavelength radial perturbations shows that the growth rate $\operatorname{Im} \omega$ of the unstable Weibel solution scales like $\Gamma_{W}$ where

$$
\begin{aligned}
\Gamma_{W}^{2} & \equiv\left[\left\langle\beta^{2}\right\rangle-\langle\beta\rangle^{2}\right] \Omega_{p}^{i 2} \\
& =\frac{\left(\beta_{e}^{2} \hat{\omega}_{p e}^{i 2}+\beta_{b}^{2} \hat{\omega}_{p b}^{i 2}\right) \hat{\omega}_{p i}^{i 2}+\left(\beta_{b}-\beta_{e}\right)^{2} \hat{\omega}_{p e}^{i 2} \hat{\omega}_{p b}^{i 2}}{\sum_{j=b, e, i} \hat{\omega}_{p j}^{i 2}}
\end{aligned}
$$

for $\beta_{i}=0$. For $\hat{\omega}_{p b}^{i 2}, \hat{\omega}_{p i}^{i 2} \ll \hat{\omega}_{p e}^{i 2}$, it follows that Eq. (28) is given to good approximation by

$$
\Gamma_{W}^{2} \simeq \beta_{e}^{2} \hat{\omega}_{p i}^{i 2}+\left(\beta_{b}-\beta_{e}\right)^{2} \hat{\omega}_{p b}^{i 2} .
$$

Note from Eq. (29) that $\Gamma_{W}$ involves the plasma frequencies of both the beam ions and the plasma ions. Focusing here on the unstable Weibel solutions for brevity, we consider the case of a cesium ion beam with $Z_{b}=1$ and $\beta_{b}=0.2$ propagating through a neutralizing background argon plasma with $Z_{i}=1, \hat{n}_{i}^{i}=(1 / 2) \hat{n}_{e}^{i}=\hat{n}_{b}^{i}$, and $\beta_{e}=0.1$ [see Eq. (26)]. Typical numerical solutions to Eq. (25) are illustrated in Figs. 8 and 9 for the choice of system parameters $r_{w}=3 r_{b}, \Omega_{p}^{o}=0$, and $\Omega_{p}^{i} r_{b} / c=1 / 3$ (Fig. 8), and $\Omega_{p}^{i} r_{b} / c=3$ (Fig. 9). Figures 8 and 9 show plots of the normalized growth rate $(\operatorname{Im} \omega) / \Gamma_{W}$ versus radial mode number $n$ and plots of the eigenfunction $\delta \hat{E}_{z}(r)$ versus $r / r_{w}$ for radial mode number $n=5$. For
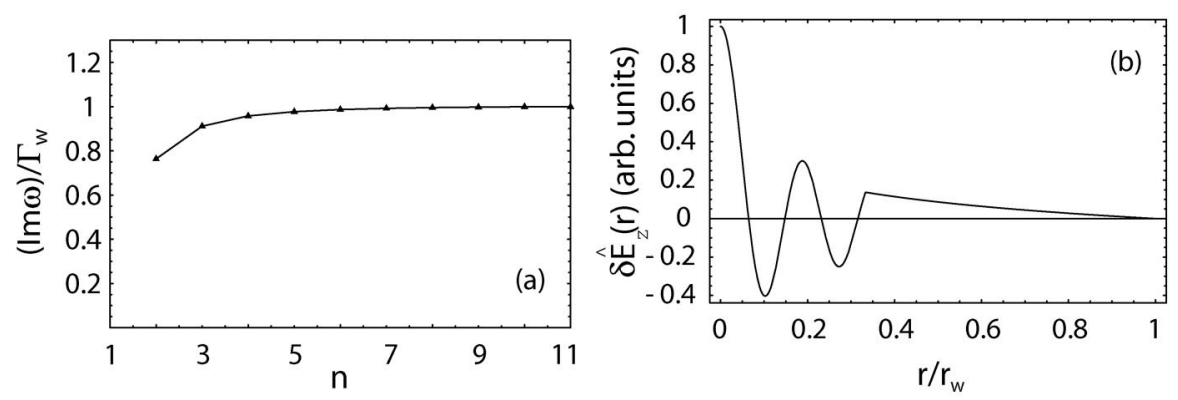

FIG. 9. Plots of (a) Weibel instability growth rate $(\operatorname{Im} \omega) / \Gamma_{W}$ versus mode number $n$, and (b) eigenfunction $\delta \hat{E}_{z}(r)$ versus $r / r_{w}$ for $n=5$ obtained from Eqs. (20) and (25). System parameters are $r_{b}=r_{w} / 3, \beta_{b}=0.2, \Omega_{p}^{i} r_{b} / c=3$, and $\Omega_{p}^{o}=0$. 
the choice of parameters in Figs. 8 and 9, note that $(\operatorname{Im} \omega)_{\max } \simeq \Gamma_{W}$ for sufficiently large $n$. As noted earlier, if current neutralization is incomplete or absent, it is expected that there will be a corresponding reduction in the Weibel instability growth rate [83] and perhaps complete stabilization in some parameter regimes. This is because of the stabilizing influence that the azimuthal self-magnetic field $B_{\theta}^{0}(r) \neq 0$ has in constraining the transverse dynamics of the beam-plasma system.

\section{Multispecies two-stream instability}

The collisionless beam-plasma configuration considered in Sec. IV B is also subject to the electrostatic twostream instability. In this case the field perturbations have electrostatic polarization with $\nabla \times \delta \mathbf{E} \simeq 0$ and $\delta \mathbf{B} \simeq 0$, and the relative streaming of the beam ions through the background plasma components provides the free energy to drive the classical two-stream instability. In this section, we make similar assumptions to those made at the beginning of Sec. IVB, including equilibrium charge neutralization and current neutralization [Eq. (19)], absence of an applied focusing field $\left(\omega_{f}=0\right)$, and the presence of a perfectly conducting cylindrical wall located at radius $r=r_{w}$. Expressing the longitudinal electric field perturbation as $\delta \mathbf{E}=-\nabla \delta \phi$, we assume axisymmetric perturbations with $\partial / \partial \theta=0$. Perturbed quantities are expressed as $\delta \phi(r, z, t)=\hat{\delta \phi}(r) \times$ $\exp \left[i\left(k_{z} z-\omega t\right)\right]$, where $k_{z}$ is the axial wave number, and $\operatorname{Im} \omega>0$ corresponds to instability (temporal growth). Without presenting algebraic details [73,84], the linearized cold-fluid-Poisson equations lead to the eigenvalue equation

$$
\begin{aligned}
\frac{1}{r} \frac{\partial}{\partial r}[ & \left.r\left(1-\sum_{j=b, e, i} \frac{\omega_{p j}^{2}(r) / \gamma_{j}^{2}}{\left(\omega-k_{z} V_{z j}\right)^{2}}\right) \frac{\partial}{\partial r} \hat{\delta \phi}\right] \\
& -k_{z}^{2}\left(1-\sum_{j=b, e, i} \frac{\omega_{p j}^{2}(r) / \gamma_{j}^{2}}{\left(\omega-k_{z} V_{z j}\right)^{2}}\right) \hat{\delta \phi}=0 .
\end{aligned}
$$

Here, $\omega_{p j}(r)=\left[4 \pi n_{j}^{0}(r) e_{j}^{2} / \gamma_{j} m_{j}\right]^{1 / 2}$ is the relativistic plasma frequency, $V_{z j}=\beta_{j} c=$ const is the average axial velocity of component $j(j=b, e, i)$, and $\gamma_{j}=$ $\left(1-\beta_{j}^{2}\right)^{-1 / 2}$ is the relativistic mass factor.

The electrostatic eigenvalue equation (30) can be solved numerically for the eigenfunction $\hat{\delta \phi}(r)$ and the complex eigenfrequency $\omega$ for a wide range of beamplasma density profiles $n_{j}^{0}(r)(j=b, e, i)$. For present purposes, we specialize again to the choice of flattop density profiles defined in Eqs. (21) and (22). In this case, the eigenfunction $\hat{\delta} \phi(r)$ can be determined analytically in the beam-plasma channel $\left(0 \leq r<r_{b}\right)$, and in the region outside the beam $\left(r_{b}<r \leq r_{w}\right)$. Employing the appropriate boundary conditions at $r=r_{b}$, and enforcing $\hat{\delta \phi}\left(r=r_{w}\right)=0$, some straightforward algebraic ma- nipulation leads to the electrostatic dispersion relation [73]

$$
\begin{aligned}
D\left(k_{z}, \omega\right)= & 1-g_{0} \sum_{j=b, e, i} \frac{\hat{\omega}_{p j}^{i 2} / \gamma_{j}^{2}}{\left(\omega-k_{z} V_{z j}\right)^{2}} \\
& -\left(1-g_{0}\right) \sum_{j=e, i} \frac{\hat{\omega}_{p j}^{o 2}}{\omega^{2}}=0 .
\end{aligned}
$$

Here, $g_{0}$ is the geometric factor defined by

$$
g_{0}=k_{z} r_{b} I_{0}^{\prime}\left(k_{z} r_{b}\right) I_{0}\left(k_{z} r_{b}\right)\left[\frac{K_{0}\left(k_{z} r_{b}\right)}{I_{0}\left(k_{z} r_{b}\right)}-\frac{K_{0}\left(k_{z} r_{w}\right)}{I_{0}\left(k_{z} r_{w}\right)}\right]
$$

for $r_{b} \neq r_{w}$. Moreover, $\hat{\omega}_{p j}^{i}=\left(4 \pi \hat{n}_{j}^{i} e_{j}^{2} / \gamma_{j} m_{j}\right)^{1 / 2}(j=$ $b, e, i)$ is the $j$ th component plasma frequency inside the beam-plasma channel $\left(0 \leq r<r_{b}\right)$, and $\hat{\omega}_{p j}^{o}=$ $\left(4 \pi \hat{n}_{j}^{o} e_{j}^{2} / m_{j}\right)^{1 / 2}(j=e, i)$ is the $j$ th component plasma frequency outside the beam-plasma channel $\left(r_{b}<r \leq\right.$ $\left.r_{w}\right)$. Similar to Sec. IV B, it is assumed that $\beta_{e}=0=$ $\beta_{i}$ in the region outside the channel, and that the plasma ions are stationary $\left(\beta_{i}=0\right)$ inside the channel. In this case, the conditions for charge neutralization and current neutralization in the beam-plasma channel reduce to Eq. (26). Finally, it should be noted from Eq. (32) that the geometric factor $g_{0}$ exhibits a strong dependence on axial wave number $k_{z}$, with

$$
\begin{aligned}
& g_{0} \simeq \frac{1}{2} k_{z}^{2} r_{b}^{2} \ln \left(\frac{r_{w}}{r_{b}}\right), \quad \text { for } k_{z}^{2} r_{w}^{2} \ll 1, \\
& g_{0} \simeq \frac{1}{2}, \quad \text { for } k_{z}^{2} r_{b}^{2} \gg 1 .
\end{aligned}
$$

Because of the geometric factors $g_{0}$ and $1-g_{0}$ in Eq. (31), the detailed properties of the two-stream instability calculated from Eq. (31) differ substantially from the infinite beam-plasma results. However, several interesting features of Eq. (31) are qualitatively evident. First, in the absence of plasma outside the beam-plasma channel $\left(\hat{\omega}_{p j}^{o 2}=0\right)$, the channel electrons undergo unstable two-stream interactions with both the beam ions and the channel plasma ions. Second, when there is plasma outside the beam-plasma channel $\left(\hat{\omega}_{p j}^{o 2} \neq 0\right)$, the channel electrons can undergo a strong unstable two-stream interaction with the plasma electrons outside the channel. Illustrative unstable solutions to the dispersion relation (31) are shown in Figs. 10 and 11 for the case where there is no plasma outside the beam-plasma channel, i.e., $\hat{n}_{e}^{o}=$ $0=\hat{n}_{i}^{o}$ for $r_{b}<r \leq r_{w}$. Here, we assume a cesium ion beam with $\beta_{b}=0.2$ and $Z_{b}=1$ propagating through background argon plasma with $Z_{i}=1$ and $\beta_{i}=0$. Assuming $\hat{n}_{b}^{i}=\hat{n}_{e}^{i} / 2=\hat{n}_{i}^{i}$, the current neutralization condition in Eq. (26) gives $\beta_{e}=0.1$. In the absence of plasma outside the beam-plasma channel, the dispersion relation (31) has two unstable branches corresponding to the interaction of the plasma electrons with the beam 

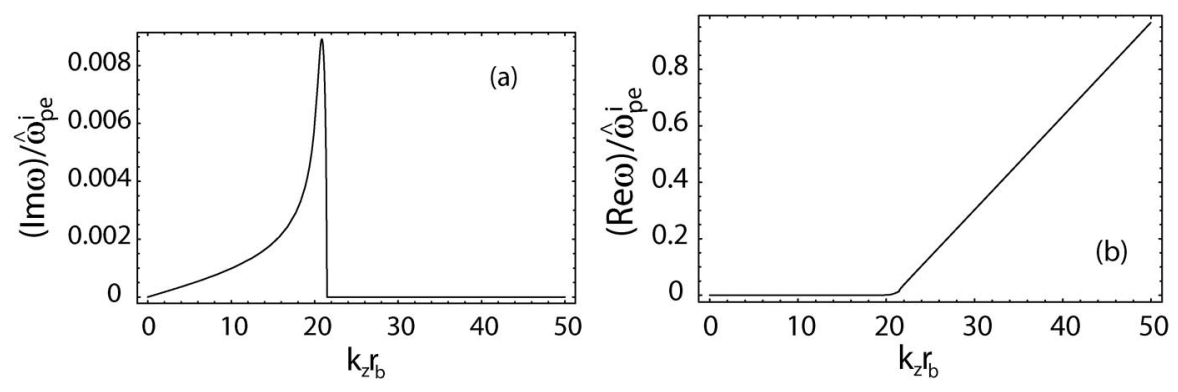

FIG. 10. Plots of (a) $(\operatorname{Im} \omega) / \hat{\omega}_{p e}^{i}$ and (b) $(\operatorname{Re} \omega) / \hat{\omega}_{p e}^{i}$ versus $k_{z} r_{b}$ calculated from the two-stream dispersion relation (31) for $r_{b}=$ $r_{w} / 3, \beta_{b}=0.2, \beta_{e}=0.1$, and $\hat{\omega}_{p e}^{i} r_{b} / c=3$ in the absence of plasma outside the beam-plasma channel.

ions, and the interaction of the plasma electrons with the plasma ions. The unstable branches in Figs. 10 and 11 correspond to the interaction of the plasma electrons with the plasma ions. Figures 10 and 11 show plots of the normalized growth $(\operatorname{Im} \omega) / \hat{\omega}_{p e}^{i}$ and real oscillation frequency $(\operatorname{Re} \omega) / \hat{\omega}_{p e}^{i}$ versus $k_{z} r_{b}$ for the two cases corresponding to $\hat{\omega}_{p e}^{i} r_{b} / c=3$ and $r_{b} / r_{w}=1 / 3$ (Fig. 10), and $\hat{\omega}_{p e}^{i} r_{b} / c=1 / 3$ and $r_{b} / r_{w}=1 / 3$ (Fig. 11). Note from Figs. 10 and 11 that the two-stream growth rate is strongly peaked as a function of $k_{z} r_{b}$. For the choice of system parameters in Fig. 10, the value of $k_{z}=k_{z m}$ at maximum growth rate satisfies $k_{z m}^{2} r_{b}^{2} \gg 1$. In this case, $g_{0}\left(k_{z m}\right) \simeq$ $1 / 2$ in Eq. (31), and the maximum growth rate $(\operatorname{Im} \omega)_{\max }$ and value of $k_{z m}$ in Fig. 10 are given to excellent approximation by the analytical estimates

$$
\begin{aligned}
(\operatorname{Im} \omega)_{\max } & \simeq\left(\frac{3}{8}\right)^{1 / 2}\left(\frac{\hat{\omega}_{p i}^{i 2}}{2 \hat{\omega}_{p e}^{i 2}}\right)^{1 / 3} \hat{\omega}_{p e}^{i} \\
\left|k_{z m}\right| r_{b} & \simeq \frac{1}{(2)^{1 / 2}} \frac{\hat{\omega}_{p e}^{i} r_{b}}{c} \frac{1}{\left|\beta_{i}-\beta_{e}\right|}
\end{aligned}
$$

where $\beta_{i}=0$ is assumed. Equation (34) pertains to the unstable plasma electron-plasma ion two-stream solution to Eq. (31). For the unstable plasma electron-beam ion solution to Eq. (31), the estimates are similar to those in Eq. (34) with $\hat{\omega}_{p i}^{i}$ replaced by $\hat{\omega}_{p b}^{i}$, and $\beta_{i}-\beta_{e}$ replaced by $\beta_{b}-\beta_{e}$.
In summary, for a cold ion beam propagating through a cold background plasma, the two-stream instability can be an important collective interaction mechanism. Since the phase velocity of the most unstable modes is close to the beam velocity $\beta_{b} c$ and the plasma ion velocity $\beta_{i} c$, modest axial velocity spreads in the beam ions and plasma ions can lead to a growth rate reduction. An important nonlinear consequence of the two-stream instability is the rapid nonlinear heating of the plasma electrons on a time scale $\tau_{\text {heat }} \sim$ a few times $(\operatorname{Im} \omega)_{\max }^{-1}$. This heating can be due to the breaking of the plasma waves.

\section{CONCLUSIONS}

This paper presented a survey of the present theoretical understanding of collective processes and beam-plasma interactions affecting intense heavy ion beam propagation in heavy ion fusion systems. In the acceleration and beam transport regions, the topics covered included (a) discussion of the conditions for quiescent beam propagation over long distances; (b) the electrostatic Harristype instability and the transverse electromagnetic Weibel-type instability in strongly anisotropic, onecomponent non-neutral ion beams (Sec. II); and (c) the dipole-mode, electron-ion two-stream instability driven by an (unwanted) component of background electrons (Sec. III). In the plasma plug and target chamber regions, collective processes associated with the interaction of the
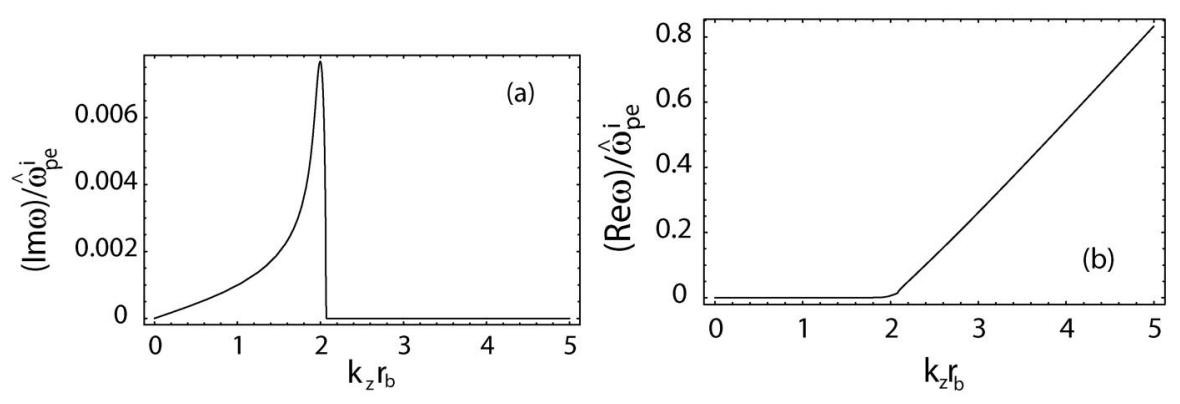

FIG. 11. Plots of (a) $(\operatorname{Im} \omega) / \hat{\omega}_{p e}^{i}$ and (b) $(\operatorname{Re} \omega) / \hat{\omega}_{p e}^{i}$ versus $k_{z} r_{b}$ calculated from the two-stream dispersion relation (31) for $r_{b}=$ $r_{w} / 3, \beta_{b}=0.2, \beta_{e}=0.1$, and $\hat{\omega}_{p e}^{i} r_{b} / c=1 / 3$ in the absence of plasma outside the beam-plasma channel. 
intense ion beam with a charge-neutralizing background plasma were described, including the electrostatic electron-ion two-stream instability, the multispecies electromagnetic Weibel instability, and the resistive hose instability (Sec. IV). Growth rate reduction mechanisms have also been identified.

\section{ACKNOWLEDGMENTS}

This research was supported by the U.S. Department of Energy.

[1] R. C. Davidson and H. Qin, Physics of Intense Charged Particle Beams in High Energy Accelerators (World Scientific, Singapore, 2001), and references therein.

[2] M. Reiser, Theory and Design of Charged Particle Beams (Wiley, New York, 1994).

[3] J. D. Lawson, The Physics of Charged-Particle Beams (Oxford Science Publications, New York, 1988).

[4] A.W. Chao, Physics of Collective Beam Instabilities in High Energy Accelerators (Wiley, New York, 1993).

[5] D. A. Edwards and M. J. Syphers, An Introduction to the Physics of High-Energy Accelerators (Wiley, New York, 1993).

[6] I. M. Kapchinskij and V.V. Vladimirskij, in Proceedings of the International Conference on High Energy Accelerators and Instrumentation, Geneva, 1959 (CERN, Geneva, 1959), p. 274.

[7] R. L. Gluckstern, in Proceedings of the 1970 Proton Linear Accelerator Conference, Batavia, $I L$, edited by M. R. Tracy (National Accelerator Laboratory, Batavia, IL, 1971), p. 811.

[8] T.-S. Wang and I. Smith, Part. Accel. 12, 247 (1982).

[9] J. Hofmann, L. J. Laslett, L. Smith, and I. Haber, Part. Accel. 13, 145 (1983).

[10] J. Struckmeier, J. Klabunde, and M. Reiser, Part. Accel. 15, 47 (1984).

[11] I. Hofmann and J. Struckmeier, Part. Accel. 21, 69 (1987).

[12] J. Struckmeier and I. Hofmann, Part. Accel. 39, 219 (1992).

[13] N. Brown and M. Reiser, Phys. Plasmas 2, 965 (1995).

[14] R. C. Davidson and H. Qin, Phys. Rev. ST Accel. Beams 2, 114401 (1999).

[15] R. L. Gluckstern, W.-H. Cheng, and H. Ye, Phys. Rev. Lett. 75, 2835 (1995).

[16] R. C. Davidson and C. Chen, Part. Accel. 59, 175 (1998).

[17] C. Chen, R. Pakter, and R. C. Davidson, Phys. Rev. Lett. 79, 225 (1997).

[18] C. Chen and R.C. Davidson, Phys. Rev. E 49, 5679 (1994).

[19] R. C. Davidson, W.W. Lee, and P. Stoltz, Phys. Plasmas 5, 279 (1998).

[20] R C. Davidson, Phys. Rev. Lett. 81, 991 (1998).

[21] R. C. Davidson, Phys. Plasmas 5, 3459 (1998).

[22] C. S. Gardner, Phys. Fluids 6, 839 (1963).

[23] P. Stoltz, R. C. Davidson, and W.W. Lee, Phys. Plasmas 6, 298 (1999).
[24] W.W. Lee, Q. Qian, and R.C. Davidson, Phys. Lett. A 230, 347 (1997).

[25] Q. Qian, W.W. Lee, and R. C. Davidson, Phys. Plasmas 4, 1915 (1997).

[26] S. I. Tzenov and R. C. Davidson, Phys. Rev. ST Accel. Beams 5, 021001 (2002).

[27] R. C. Davidson and H. Qin, Phys. Rev. ST Accel. Beams 4, 104401 (2001).

[28] R. C. Davidson, H. Qin, and P. J. Channell, Phys. Rev. ST Accel. Beams 2, 074401 (1999); 3, 029901 (2000).

[29] R.C. Davidson et al., Laser Part. Beams 20, 377 (2002).

[30] E. G. Harris, Phys. Rev. Lett. 2, 34 (1959).

[31] E. A. Startsev, R. C. Davidson, and H. Qin, Phys. Plasmas 9, 3138 (2002).

[32] E. A. Startsev, R. C. Davidson, and H. Qin, Laser Part. Beams 20, 585 (2002).

[33] E. A. Startsev, R. C. Davidson, and H. Qin, Phys. Rev. ST Accel. Beams 6, 084401 (2003).

[34] R. A. Kishek, P. G. O'Shea, and M. Reiser, Phys. Rev. Lett. 85, 4514 (2000).

[35] J. Haber, A. Friedman, D. P. Grote, S. M. Lund, and R. A. Kishek, Phys. Plasmas 6, 2254 (1999).

[36] A. Friedman, D. P. Grote, and I. Haber, Phys. Fluids B 4, 2203 (1992).

[37] E. S. Weibel, Phys. Rev. Lett. 2, 83 (1959).

[38] E. A. Startsev and R. C. Davidson, Phys. Plasmas 10, 4829 (2003).

[39] R.C. Davidson, D. A. Hammer, I. Haber, and C.E. Wagner, Phys. Fluids 15, 317 (1972).

[40] R. Lee and M. Lampe, Phys. Rev. Lett. 31, 1390 (1973).

[41] C. A. Kapetanakos, Appl. Phys. Lett. 25, 484 (1974).

[42] M. Honda, J. Meyer-ter-Vehn, and A. Pukhov, Phys. Rev. Lett. 85, 2128 (2000).

[43] V. K. Neil and A. M. Sessler, Rev. Sci. Instrum. 36, 429 (1965).

[44] E. P. Lee, in Proceedings of the 1981 Linear Accelerator Conference, Washington, DC (Los Alamos National Laboratory Report No. LA-9234-C), pp. 263-265.

[45] E. P. Lee, Part. Accel. 37, 307 (1992).

[46] D. G. Koshkarev and P. R. Zenkevich, Part. Accel. 3, 1 (1972).

[47] E. Keil. and B. Zotter, Report No. CERN-ISR-TH-71-58, 1971.

[48] L. J. Laslett, A. M. Sessler, and D. Moehl, Nucl. Instrum. Methods 121, 517 (1974).

[49] R. C. Davidson, H. Qin, and G. Shvets, Phys. Rev. ST Accel. Beams 6, 104402 (2003).

[50] D. Neuffer, B. Colton, D. Fitzgerald, T. Hardek, R. Hutson, R. Macek, M. Plum, H. Thiessen, and T.S. Wang, Nucl. Instrum. Methods Phys. Res., Sect. A 321, 1 (1992).

[51] R. J. Macek et al., in Proceedings of the 2001 Particle Accelerator Conference, Chicago (IEEE, Piscataway, NJ, 2001), Vol. 1, p. 688.

[52] R. C. Davidson, H. Qin, and T.-S. Wang, Phys. Lett. A 252, 213 (1999).

[53] R. C. Davidson, H. Qin, P. H. Stoltz, and T.-S. Wang, Phys. Rev. ST Accel. Beams 2, 054401 (1999).

[54] R. C. Davidson and H. Qin, Phys. Lett. A 270, 177 (2000). 
[55] H. Qin, R. C. Davidson, and W.W. Lee, Phys. Rev. ST Accel. Beams 3, 084401 (2000); 3, 109901 (2000).

[56] R. C. Davidson and H.S. Uhm, Phys. Lett. A 285, 88 (2001).

[57] H. Qin, R. C. Davidson, E. A. Startsev, and W.W. Lee, Laser Part. Beams 21, 21 (2003).

[58] T.-S. Wang, P. J. Channell, R. J. Macek, and R. C. Davidson, Phys. Rev. ST Accel. Beams 6, 014204 (2003).

[59] H. Qin, E. A. Startsev, and R. C. Davidson, Phys. Rev. ST Accel. Beams 6, 014401 (2003).

[60] H. Qin, Phys. Plasmas 10, 2078 (2003).

[61] E. P. Lee, Phys. Fluids 21, 1327 (1978).

[62] E. J. Lauer, R. J. Briggs, T. J. Fesendon, R. E. Hester, and E. P. Lee, Phys. Fluids 21, 1344 (1978).

[63] M. N. Rosenbluth, Phys. Fluids 3, 932 (1960).

[64] H.S. Uhm and M. Lampe, Phys. Fluids 23, 1574 (1980).

[65] H. S. Uhm and M. Lampe, Phys. Fluids 24, 1553 (1981).

[66] R. F. Fernsler, S. P. Slinker, M. Lampe, and R. F. Hubbard, Phys. Plasmas 2, 4338 (1995), and references therein.

[67] H. S. Uhm and R. C. Davidson, Phys. Rev. ST Accel. Beams 6, 034204 (2003).

[68] H.S. Uhm, R. C. Davidson, and I. D. Kaganovich, Phys. Plasmas 8, 4637 (2001).

[69] G. Joyce and M. Lampe, Phys. Fluids 26, 3377 (1983).

[70] H.S. Uhm and R. C. Davidson, Phys. Fluids 23, 1586 (1980).

[71] D. V. Rose, T. C. Genoni, D. R. Welch, and C. L. Olson, in Proceedings of the 2003 Particle Accelerator Conference, Portland, OR (IEEE, Piscataway, NJ, 2003), p. 3165.
[72] D. R. Welch, D.V. Rose, S. S. Yu, and G. L. Olson, in Proceedings of the 2003 Particle Accelerator Conference, Portland, OR (Ref. [71]), p. 2685.

[73] R. C. Davidson, I. Kaganovich, and E. A. Startsev, Weibel and Two-Stream Instabilities for Intense Charged Particle Beam Propagation Through Neutralizinig Background Plasma, Princeton Plasma Physics Laboratory Report No. PPPL-3940, 2004.

[74] I. D. Kaganovich, G. Shvets, E. Startsev, and R. C. Davidson, Phys. Plasmas 8, 4180 (2001).

[75] I. Kaganovich, E. A. Startsev, and R. C. Davidson, Laser Part. Beams 20, 497 (2002).

[76] D.V. Rose, P. F. Ottinger, D. R. Welch, B. V. Oliver, and C. L. Olson, Phys. Plasmas 6, 4094 (1999).

[77] D. R. Welch, D. V. Rose, B.V. Oliver, T. C. Genoni, C. L. Olson, and S. S. Yu, Phys. Plasmas 9, 2344 (2002).

[78] D. R. Welch, D. V. Rose, W. M. Sharp, C. L. Olson, and S. S. Yu, Laser Part. Beams 20, 621 (2002).

[79] C.S. Debonnel, D. R. Welch, D. V. Rose, S. S. Yu, and P. F. Peterson, Fusion Sci. Technol. 43, 408 (2003).

[80] D.V. Rose, D. R. Welch, C. L. Olson, S. S. Yu, S. Neff, W. M. Sharp, and the ARIES Team, Fusion Sci. Technol. (to be published).

[81] S. S. Yu, W. R. Meier, R. P. Abbott, J. J. Barnard, T. Brown, D. A. Callahan, P. Heitzenroeter, J. F. Latowski, B. G. Logan, S. J. Pemberton, P. F. Peterson, D. V. Rose, G.-L. Sabbi, W. M. Sharp, and D. R. Welch, Fusion Sci. Technol. 44, 266 (2003).

[82] See also Chap. 4 and Sec. 8.6 of Ref. [1].

[83] R. C. Davidson, Physics of Nonneutral Plasmas (World Scientific, Singapore, 2001), pp. 272-276.

[84] See, for example, Sec. 9.5 of Ref. [1]. 